\title{
Distributed resource allocation for MISO downlink systems via the alternating direction method of multipliers
}

\author{
Satya Krishna Joshi*, Marian Codreanu and Matti Latva-aho
}

\begin{abstract}
We provide distributed algorithms for the radio resource allocation problem in multicell downlink multi-input single-output systems. Specifically, the problems of (1) minimizing total transmit power subject to signal-to-interference-plus-noise ratio (SINR) constraints of each user and (2) SINR balancing subject to total transmit power constraints are considered. We propose a consensus-based distributed algorithm and the fast solution method via alternating the direction method of multipliers. First, we derive a distributed algorithm for minimization of total transmit power. Then, in conjunction with the bracketing method, a distributed algorithm for SINR balancing is derived. Numerical results show that the proposed distributed algorithms achieve the optimal centralized solution.
\end{abstract}

Keywords: Distributed optimization; Multicell networks; Minimum power beamforming; Signal-to-interference-plus-noise ratio (SINR) balancing; Alternating direction method of multipliers (ADMM); Dual decomposition; Second-order cone program (SOCP)

\section{Introduction}

We provide distributed algorithms for the problem of resource allocation for multicell downlink systems with linear precoding. The base stations (BSs) are assumed to have multiple antennas while all the users are equipped with single antenna. Full channel state information is assumed to be available at both the BSs and the users, and all the users share the same frequency bandwidth. Under this setting, we consider the following two optimization problems: P1 - minimization of the total transmission power subject to minimum signal-to-interference-plusnoise ratio (SINR) constraints of each user, and P2 - SINR balancing subject to total transmit power constraint of BSs.

Several centralized algorithms for problems P1 and P2 have been proposed in the literature. See, e.g., [1-6] for problem P1 and [7-10] for problem P2. Unfortunately, the centralized method is not practical for the resource allocation due to high overhead required for collecting all channel state information at the central processing

*Correspondence: sjoshi@ee.oulu.fi

Centre for Wireless Communications, University of Oulu, P.O. Box 4500, Oulu Fl-90014, Finland unit. Therefore, to share the workload of the central controller and to overcome impelling backhaul overhead, the distributed algorithm is desirable in practice.

Distributed methods for problem P1 for multiple-input and single-output (MISO) multicell wireless systems have been proposed in [11-15]. The algorithm in [11] is based on uplink-downlink duality, where the minimum power downlink beamformers designing problem is solved using a dual uplink problem. The algorithm in [11] is a multicell generalization of the algorithm proposed in [16] for the single-cell case. In [12] dual decomposition method is adopted, and the algorithm in [13] is based on primal decomposition. Both in primal and dual decomposition methods [17], the master problem is solved using an iterative method such as the subgradient method [18]. Even though problem P1 is a convex problem and can be easily solved via primal or dual decomposition (see, $[12,13]$ ), the convergence speed of those algorithms are slow and highly sensitive on the subgradient step size [18]. A game theoretic approach is considered in [14].

Problem P2 is a quasiconvex problem [16]. Thus, the centralized method based on bisection search [19] is commonly used, e.g., $[10,16]$. Combining the bisection search and the uplink-downlink SINR duality, a distributed

\section{望 Springer}

(c) 2014 Joshi et al.: licensee Springer. This is an Open Access article distributed under the terms of the Creative Commons Attribution License (http://creativecommons.org/licenses/by/2.0), which permits unrestricted use, distribution, and reproduction in any medium, provided the original work is properly cited. 
algorithm is proposed in [20]. The algorithm in [20] is a hierarchical iterative algorithm which consists of outer and inner iterations, where the bisection search is carried out in the outer iteration and uplink-downlink SINR duality is used for the inner iteration.

The alternating direction method of multipliers (ADMM) is a simple but powerful algorithm that is well suited to distributed convex optimization. ADMM combines the benefits of dual decomposition and augmented Lagrangian methods [21]. Hence, ADMM is numerically stable than dual decomposition and hence suitable for many practical optimization problem [21]. Due to superior stability properties and decomposability, ADMM has been applied to a wide rage of applications, such as compressed sensing [22], image restoration [23], signal processing [15,24,25], etc.; see [21] for the recent survey. In many applications, the ADMM method has been observed to converge fast $[21,26,27]$; and when the objective function is strongly convex and Lipschitz continuous, ADMM can even guarantee the liner rate of convergence $[28,29]$.

The main contribution of the paper is to propose consensus-based distributed algorithms for problems P1 and P2, and the fast solution method via ADMM [21]. The ADMM turns the original problem into a series of iterative steps, namely, local variable update, global variable update, and dual variable update [21]. The local variable and dual variable updates are carried out independently in parallel by all BSs, while the global variable update is carried out by BS coordination. In this paper, we first derive distributed algorithm for problem P1. Then, we extend the formulation of problem P1 to derive the distributed algorithm for problem P2. In particular, we recast the problem into a more tractable form and combine bracketing method (e.g., golden ratio search) [30,31] with ADMM to derive the distributed algorithm for problem P2.

Recently, for problem P1, by considering the uncertainty in the channel measurements, an algorithm based on ADMM has been proposed in [15]. In our paper, we consider perfect channel state information (CSI) and use the consensus technique to solve the problem. Then, we apply ADMM to derive the distributed algorithm. The consensus technique can be easily decomposed into a set of subproblems suitable for distributed implementation $[21,24]$. Hence, the algorithm formulation in this paper is more intuitive than that provided in [15]. It is worth noting that this paper extends our recent work [32] to SINR balancing problem (i.e., problem P2). In addition, for problem P1, we show that the proposed distributed algorithm converges to the optimal centralized solution. Moreover, for problem P1, we also provide a method to find the ADMM penalty parameter that leads faster convergence of the algorithm.

Note that problem P2 is a quasiconvex problem. To the best of our knowledge there is no convergence theory to the ADMM method for a quasiconvex problem. However, if each step of the ADMM iteration is tractable, the ADMM algorithm can still be used to derive (possibly suboptimal) distributed methods for problem P2 [21, Chapter 9]. Though these methods are not provably optimal, our numerical results show that the proposed algorithm converged to the optimal solution in all simulated cases.

The remainder of this paper is organized as follows. The considered MISO system model and problem formulation are described in Section 2. The distributed algorithm for sum power minimization (P1) is derived in Section 3. Next, in Section 4, we derive the distributed algorithm for SINR balancing problem (P2). The numerical results are presented in Section 5, and Section 6 concludes our paper.

Notations: All boldface lower case and upper case letters represent vectors and matrices, respectively, and calligraphy letters represent sets. The notation $\mathbb{C}^{T}$ denotes the set of complex $T$-vectors, $|x|$ denotes the absolute value of the scalar $x,\|\mathbf{x}\|_{2}$ denotes the Euclidean norm of the vector $\mathbf{x}$, I denotes the identity matrix, and $\mathcal{C N}(\mathbf{m}, \mathbf{C})$ denotes the complex circular symmetric Gaussian vector distribution with mean $\mathbf{m}$ and covariance matrix $\mathbf{C}$. The superscripts $(\cdot)^{\mathrm{H}}$ and $(\cdot)^{\star}$ are used to denote a Hermitian transpose of a matrix and a solution of an optimization problem, respectively.

\section{System model and problem formulation}

A multicell MISO downlink system, with $N$ BSs each equipped with $T$ transmit antennas, is considered. The set of all BSs is denoted by $\mathcal{N}$, and we label them with the integer values $n=1, \ldots, N$. The transmission region of each $\mathrm{BS}$ is modeled as a disc with radius $R_{\mathrm{BS}}$ centered at the location of the BS. Single data stream is transmitted for each user. We denote the set of all data streams in the system by $\mathcal{L}$, and we label them with the integer values $l=1, \ldots, L$. The transmitter node (i.e., the BS) of $l$ th data stream is denoted by $\operatorname{tran}(l)$, and the receiver node of $l$ th data stream is denoted by $\operatorname{rec}(l)$. We have $\mathcal{L}=\cup_{n \in \mathcal{N}} \mathcal{L}(n)$, where $\mathcal{L}(n)$ denotes the set of data streams transmitted by $n$th BS. Note that the intended users of the data streams transmitted by each BS are necessarily located inside the transmission region of the BS (see Figure 1).

The antenna signal vector transmitted by $n$th BS is given by

$$
\mathbf{x}_{n}=\sum_{l \in \mathcal{L}(n)} d_{l} \mathbf{m}_{l}
$$

where $d_{l} \in \mathbb{C}$ and $\mathbf{m}_{l} \in \mathbb{C}^{T}$ represent the information symbol and the transmit beamformer associated to $l$ th data stream, respectively. We assume that $d_{l}$ is normalized such that $\mathrm{E}\left|d_{l}\right|^{2}=1$. Moreover, we assume that the data streams are independent, i.e., $\mathrm{E}\left\{d_{l} d_{j}^{*}\right\}=0$ for all $l \neq j$, where $l, j \in \mathcal{L}$. 


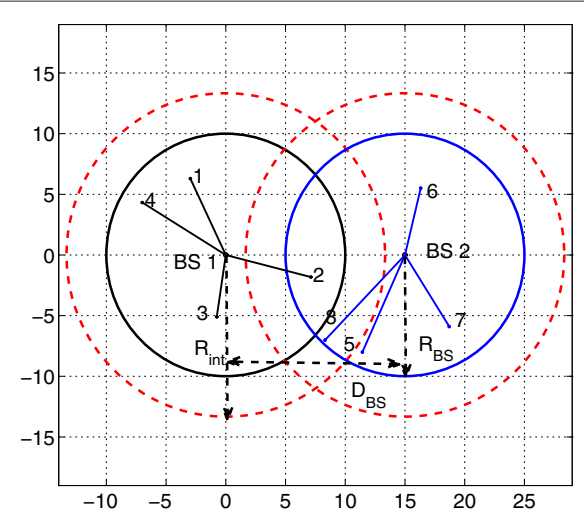

(a)

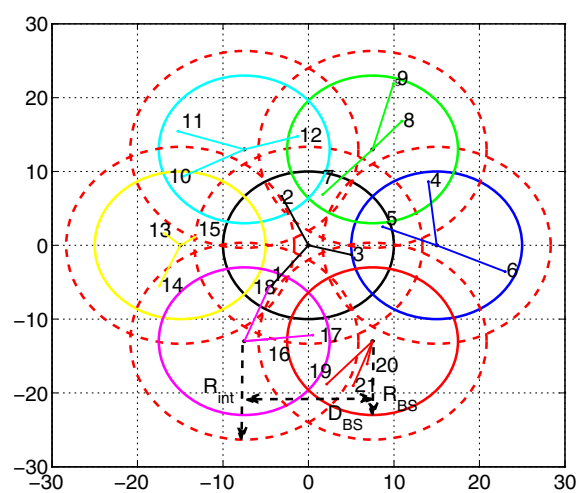

(b)

Figure 1 Multicell networks. (a) Multicell network 1 , where $\mathcal{N}=\{1,2\}, \mathcal{L}(1)=\{1,2,3,4\}, \mathcal{L}(2)=\{5,6,7,8\}$, and $\mathcal{L}_{\text {int }}=\{2,8\}$; (b) Multicell network 2, where $\mathcal{N}=\{1,2,3,4,5,6,7\}, \mathcal{L}(1)=\{1,2,3\}, \mathcal{L}(2)=\{4,5,6\}, \mathcal{L}(3)=\{7,8,9\}, \mathcal{L}(4)=\{10,11,12\}, \mathcal{L}(5)=\{13,14,15\}$, $\mathcal{L}(6)=\{16,17,18\}, \mathcal{L}(7)=\{19,20,21\}, \mathcal{L}_{\text {int }}=\{1,2,3,4,5,7,10,12,14,15,16,17,18,19\}$.

The signal received at $\operatorname{rec}(l)$ can be expressed as

$$
\begin{aligned}
y_{l}=d_{l} \mathbf{h}_{l l}^{\mathrm{H}} \mathbf{m}_{l}+ & \sum_{j \in \mathcal{L}(\operatorname{tran}(l)), j \neq l} d_{j} \mathbf{h}_{j l}^{\mathrm{H}} \mathbf{m}_{j} \\
& (\text { intra-cell interference) } \\
& +\sum_{n \in \mathcal{N} \backslash\{\operatorname{tran}(l)\}} \sum_{j \in \mathcal{L}(n)} d_{j} \mathbf{h}_{j l}^{\mathrm{H}} \mathbf{m}_{j}+n_{l},
\end{aligned}
$$

(out-of-cell interference)

where $\mathbf{h}_{j l}^{\mathrm{H}} \in \mathbb{C}^{1 \times T}$ is the channel matrix between $\operatorname{tran}(j)$ and $\operatorname{rec}(l)$, and $n_{l}$ is circular symmetric complex gaussian noise with variance $\sigma_{l}^{2}$. Note that the second right hand term in (2) represents the intra-cell interference and the third right hand term represents the out-of-cell interference. The received SINR of $l$ th data stream is given by

$$
\Gamma_{l}=\frac{\left|\mathbf{h}_{l l}^{\mathrm{H}} \mathbf{m}_{l}\right|^{2}}{\sigma_{l}^{2}+\sum_{j \in \mathcal{L}(\operatorname{tran}(l)), j \neq l}\left|\mathbf{h}_{j l}^{\mathrm{H}} \mathbf{m}_{j}\right|^{2}+\sum_{n \in \mathcal{N} \backslash\{\operatorname{tran}(l)\}} z_{n l}^{2}},
$$

where $z_{n l}^{2}=\sum_{j \in \mathcal{L}(n)}\left|\mathbf{h}_{j l}^{\mathrm{H}} \mathbf{m}_{j}\right|^{2}$ represents the power of the out-of-cell interference from $n$th BS to $\operatorname{rec}(l)$.

The out-of-cell interference term in (3) (i.e., $\left.\sum_{n \in \mathcal{N} \backslash\{\operatorname{tran}(l)\}} z_{n l}^{2}\right) \quad$ prevents resource allocation on an intra-cell basis and demands BS cooperation/coordination. To avoid unnecessary coordination between far apart-located BSs, we make the following assumption: transmission from $n$th BS interfere the $l$ th data stream (transmitted by BS $b \neq n$ ) only if the distance between $n$th BS and $\operatorname{rec}(l)$ is smaller than a threshold $R_{\text {int }}{ }^{a}$. The disc with radius $R_{\text {int }}$ centered at the location of any BS is referred to as the interference region of the BS (see Figure 1). Thus, if $n$th BS located at a distance larger than $R_{\text {int }}$ to $\operatorname{rec}(l)$, the associated $z_{n l}$ components are set to zero ${ }^{\mathrm{b}}$. Based on the assumption above, we can express $\Gamma_{l}$ as

$$
\Gamma_{l}=\frac{\left|\mathbf{h}_{l l}^{\mathrm{H}} \mathbf{m}_{l}\right|^{2}}{\sigma_{l}^{2}+\sum_{j \in \mathcal{L}(\operatorname{tran}(l)), j \neq l}\left|\mathbf{h}_{j l}^{\mathrm{H}} \mathbf{m}_{j}\right|^{2}+\sum_{n \in \mathcal{N}_{\text {int }}(l)} z_{n l}^{2}},
$$

where $\mathcal{N}_{\text {int }}(l) \subseteq \mathcal{N} \backslash\{\operatorname{tran}(l)\}$ is the set of out-of-cell interfering BSs that are located at a distance less than $R_{\text {int }}$ to $\operatorname{rec}(l)$. For example, in Figure 1a, we have $\mathcal{N}_{\text {int }}(2)=\{2\}$, $\mathcal{N}_{\text {int }}(8)=\{1\}$, and $\mathcal{N}_{\text {int }}(l)=\emptyset$ for all $l \in\{1,3,4,5,6,7\}$. Moreover, it is useful to define the set $\mathcal{L}_{\text {int }}$ of all data streams that are subject to the out-of-cell interference, i.e., $\mathcal{L}_{\text {int }}=\left\{l \mid l \in \mathcal{L}, \mathcal{N}_{\text {int }}(l) \neq \emptyset\right\}$. For example, in Figure 1a, we have $\mathcal{L}_{\text {int }}=\{2,8\}$.

The total transmitted power of the multicell downlink system can be expressed as

$$
P=\sum_{n \in \mathcal{N}} \sum_{l \in \mathcal{L}(n)}\left\|\mathbf{m}_{l}\right\|_{2}^{2} .
$$

Assuming that the SINR $\Gamma_{l}$ is subject to the constraint $\Gamma_{l} \geq \gamma_{l}$ for each user $l \in \mathcal{L}$, the problem of minimizing the total transmitted power (i.e., P1) can be expressed as

$$
\begin{aligned}
\text { P1: minimize } & \sum_{n \in \mathcal{N}} \sum_{l \in \mathcal{L}(n)}\left\|\mathbf{m}_{l}\right\|_{2}^{2} \\
\text { subject to } & \frac{\left|\mathbf{h}_{l l}^{\mathrm{H}} \mathbf{m}_{l}\right|^{2}}{\sigma_{l}^{2}+\sum_{j \in \mathcal{L}(\operatorname{tran}(l)), j \neq l}\left|\mathbf{h}_{j l}^{\mathrm{H}} \mathbf{m}_{j}\right|^{2}+\sum_{n \in \mathcal{N}_{\text {int }}(l)} z_{n l}^{2}} \geq \gamma_{l}, \\
& l \in \mathcal{L} \\
& z_{n l}^{2}=\sum_{j \in \mathcal{L}(n)}\left|\mathbf{h}_{j l}^{\mathrm{H}} \mathbf{m}_{j}\right|^{2}, \quad l \in \mathcal{L}_{\text {int }}, n \in \mathcal{N}_{\text {int }}(l),
\end{aligned}
$$

with variables $\left\{\mathbf{m}_{l}\right\}_{l \in \mathcal{L}}$ and $\left\{z_{n l}\right\}_{l \in \mathcal{L}_{\text {int }}, n \in \mathcal{N}_{\text {int }}(l)}$.

Providing fairness among the users with per BS power constraint (i.e., $\sum_{j \in \mathcal{L}(n)}\left\|\mathbf{m}_{l}\right\|_{2}^{2} \leq p_{n}^{\max }$ ) is another important resource allocation problem. One way ${ }^{\mathrm{d}}$ of providing 
fairness among the users is by maximizing the minimum SINR (i.e., P2) [16], which can be formulated as

P2 : maximize $\min _{l \in \mathcal{L}}\left(\frac{\left|\mathbf{h}_{l l}^{\mathrm{H}} \mathbf{m}_{l}\right|^{2}}{\sigma_{l}^{2}+\sum_{j \in \mathcal{L}(\operatorname{tran}(l)), j \neq l}\left|\mathbf{h}_{j l}^{\mathrm{H}} \mathbf{m}_{j}\right|^{2}+\sum_{n \in \mathcal{N} \text { int }(l)} z_{n l}^{2}}\right)$ subject to $z_{n l}^{2}=\sum_{j \in \mathcal{L}(n)}\left|\mathbf{h}_{j l}^{\mathrm{H}} \mathbf{m}_{j}\right|^{2}, \quad l \in \mathcal{L}_{\text {int }}, n \in \mathcal{N}_{\text {int }}(l)$

$$
\sum_{j \in \mathcal{L}(n)}\left\|\mathbf{m}_{l}\right\|_{2}^{2} \leq p_{n}^{\max }, \quad n \in \mathcal{N}
$$

where the variables are $\left\{\mathbf{m}_{l}\right\}_{l \in \mathcal{L}}$ and $\left\{z_{n l}\right\}_{l \in \mathcal{L}_{\text {int }}, n \in \mathcal{N}_{\text {int }}(l)}$. Finally, to improve the readability of the paper, we summarize a list of sets used in this paper in Table 1.

\section{Sum power minimization}

In this section, we derive a distributed algorithm for problem (4), i.e., P1. First, we equivalently reformulate the original problem (4) in a form of global consensus problem. Then, we derive the proposed distributed algorithm based on ADMM [21].

\subsection{An equivalent reformulation: sum power minimization}

We start by reformulating sum power minimization problem (4) as

$$
\begin{aligned}
\operatorname{minimize} & \sum_{n \in \mathcal{N}} \sum_{l \in \mathcal{L}(n)}\left\|\mathbf{m}_{l}\right\|_{2}^{2} \\
\text { subject to } & \frac{\left|\mathbf{h}_{l l}^{\mathrm{H}} \mathbf{m}_{l}\right|^{2}}{\sigma_{l}^{2}+\sum_{j \in \mathcal{L}(\operatorname{tran}(l)), j \neq l}\left|\mathbf{h}_{j l}^{\mathrm{H}} \mathbf{m}_{j}\right|^{2}+\sum_{n \in \mathcal{N}_{\text {int }}(l)} z_{n l}^{2}} \geq \gamma_{l}, \\
& l \in \mathcal{L} \\
& z_{n l}^{2} \geq \sum_{j \in \mathcal{L}(n)}\left|\mathbf{h}_{j l}^{\mathrm{H}} \mathbf{m}_{j}\right|^{2}, \quad l \in \mathcal{L}_{\text {int }}, n \in \mathcal{N}_{\text {int }}(l),
\end{aligned}
$$

\section{Table 1 Summary of a list of sets}

\begin{tabular}{ll}
\hline Set & Description \\
\hline $\mathcal{N}$ & Set of all BSs \\
$\mathcal{L}$ & Set of all data streams \\
$\mathcal{L}(n)$ & Set of data streams transmitted by $n$th BS \\
$\mathcal{N}_{\text {int }}(l)$ & Set of out-of-cell BSs interfering to /th data stream \\
$\mathcal{L}_{\text {int }}$ & Set of all data streams that are subject to the out-of-cell \\
& interference \\
$\mathcal{I}_{\text {int }}(n)$ & Set of links for which BS $n$ acts as the out-of-cell interferer \\
$\mathcal{L}_{\text {int }}(n)$ & Set of links in BS $n$ that are affected by the out-of-cell \\
& interference \\
\hline
\end{tabular}

where the variables are $\left\{\mathbf{m}_{l}\right\}_{l \in \mathcal{L}}$ and $\left\{z_{n l}\right\}_{l \in \mathcal{L}_{\text {int }}, n \in \mathcal{N}_{\text {int }}(l)}$. Problems (4) and (6) are equivalent as it can be easily shown (e.g., by contradiction) that the second inequality holds with equality at the optimal point.

Recall that $z_{n l}^{2}$ in problem (6) represents power of the out-of-cell interference caused by $n$th BS at $\operatorname{rec}(l)$, and hence, variable $z_{n l}$ couples exactly two BSs (i.e., BS $n$ and BS $\operatorname{tran}(l))$. We use consensus technique to distribute problem (6) over the BSs. The method consist of introducing local copies of the coupling variables $z_{n l}$ for all $l \in \mathcal{L}_{\text {int }}$, $n \in \mathcal{N}_{\text {int }}(l)$ at each BS (see Figure 2).

Let us define $x_{k, n l}$ as the local copy of $z_{n l}$ at BS $k$. Thus for each $z_{n l}$, we make two local copies, i.e., $x_{n, n l}$ at BS $n$ and $x_{\operatorname{tran}(l), n l}$ at BS $\operatorname{tran}(l)$. Then, problem (6) can be written equivalently in a global consensus form as

$$
\begin{array}{ll}
\text { minimize } & \sum_{n \in \mathcal{N}} \sum_{l \in \mathcal{L}(n)}\left\|\mathbf{m}_{l}\right\|_{2}^{2} \\
\text { subject to } & \frac{\left|\mathbf{h}_{l l}^{\mathrm{H}} \mathbf{m}_{l}\right|^{2}}{\sigma_{l}^{2}+\sum_{j \in \mathcal{L}(\operatorname{tran}(l)), j \neq l}\left|\mathbf{h}_{j l}^{\mathrm{H}} \mathbf{m}_{j}\right|^{2}+\sum_{b \in \mathcal{N}_{\text {int }}(l)} x_{n, b l}^{2}} \geq \gamma_{l}, \\
& n \in \mathcal{N}, l \in \mathcal{L}(n) \\
& x_{n, n l}^{2} \geq \sum_{j \in \mathcal{L}(n)}\left|\mathbf{h}_{j l}^{\mathrm{H}} \mathbf{m}_{j}\right|^{2}, \quad l \in \mathcal{L}_{\text {int }}, n \in \mathcal{N}_{\text {int }}(l) \\
x_{k, n l} & =z_{n l}, \quad k \in\{n, \operatorname{tran}(l)\}, l \in \mathcal{L}_{\text {int }}, n \in \mathcal{N}_{\text {int }}(l),
\end{array}
$$

with variables $\left\{\mathbf{m}_{l}\right\}_{l \in \mathcal{L}},\left\{x_{k, n l}\right\}_{k \in\{n, \operatorname{tran}(l)\}, l \in \mathcal{L}_{\text {int }}, n \in \mathcal{N}_{\text {int }}(l)}$, and $\left\{z_{n l}\right\}_{l \in \mathcal{L}_{\text {int }}, n \in \mathcal{N}_{\text {int }}(l)}$. Note that in the SINR constraints of problem (7), we have replaced $z_{b l}$ by the local copy $x_{n, b l}$ and used $\mathcal{L}=\cup_{n \in \mathcal{N}} \mathcal{L}(n)$. In the second inequality constraints of (7), we have replaced $z_{n l}$ by the local copy $x_{n, n l}$. The last set of equality constraints of (7) are called consistency constraints, and they enforce the local copies $\left\{x_{k, n l}\right\}_{k \in\{n, \operatorname{tran}(l)\}}$ to be equal to the corresponding global variable $z_{n l}$.

Problem (7) is not a convex problem. However, by following the approach of [16, Section IV-B], problem (7) can be equivalently cast in the form of convex problem. To do this, let us define the matrix $\mathbf{M}_{n}=\left[\mathbf{m}_{l}\right]_{l \in \mathcal{L}(n)}$ obtained by concatenating the column vectors $\mathbf{m}_{l}$. Then, by following the approach of $[16$, Section IV-B], problem (7) can be equivalently reformulated in the form of convex problem as

$$
\begin{aligned}
\operatorname{minimize} & \sum_{n \in \mathcal{N}} \sum_{l \in \mathcal{L}(n)}\left\|\mathbf{m}_{l}\right\|_{2}^{2} \\
\text { subject to } & {\left[\begin{array}{c}
\sqrt{1+\frac{1}{\gamma_{l}}} \mathbf{h}_{l l}^{\mathrm{H}} \mathbf{m}_{l} \\
\mathbf{M}_{n}^{\mathrm{H}} \mathbf{h}_{l l} \\
\tilde{\mathbf{x}}_{l} \\
\sigma_{l}
\end{array}\right] \succeq_{\mathrm{SOC}} 0, \quad n \in \mathcal{N}, l \in \mathcal{L}(n) } \\
& {\left[\begin{array}{c}
x_{n, n l} \\
\mathbf{M}_{n}^{\mathrm{H}} \mathbf{h}_{j l}
\end{array}\right] \succeq_{\mathrm{SOC}} 0, \quad l \in \mathcal{L}_{\text {int }}, n \in \mathcal{N}_{\text {int }}(l) } \\
& x_{k, n l}=z_{n l}, \quad k \in\{n, \operatorname{tran}(l)\}, l \in \mathcal{L}_{\text {int }}, n \in \mathcal{N}_{\text {int }}(l),
\end{aligned}
$$




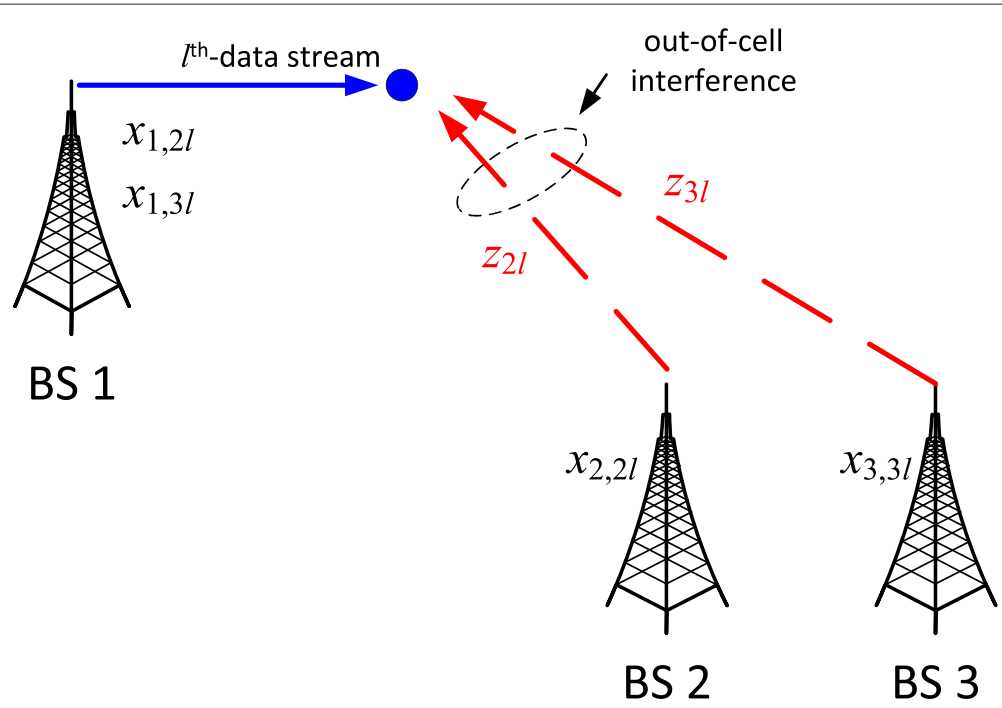

Figure 2 Illustration of BS coupling, and introducing local copies to decouple a problem. BS 2 and BS 3 are coupled with BS 1 due to coupling variables $z_{2 l}$ and $z_{3 /}$, respectively. To distribute the problem, local copy $x_{1,2 l}$ of $z_{2 /}$ at BS 1 and local copy $x_{2,2 l}$ of $z_{2 l}$ at BS 2 are introduced. Similarly, local copy $x_{1,3 /}$ of $z_{31}$ at BS 1 and local copy $x_{3,31}$ of $z_{3 /}$ at BS 3 are introduced.

with variables $\left\{\mathbf{M}_{n}\right\}_{n \in \mathcal{N}},\left\{x_{k, n l}\right\}_{k \in\{n, \operatorname{tran}(l)\}, l \in \mathcal{L}_{\text {int }}, n \in \mathcal{N}_{\text {int }}(l)}$, and $\left\{z_{n l}\right\}_{l \in \mathcal{L}_{\text {int }}, n \in \mathcal{N}_{\text {int }}(l)}$, where vector $\tilde{\mathbf{x}}_{l}=\left\{x_{n, b l}\right\}_{b \in \mathcal{N}_{\text {int }}(l)}$, the matrix $\mathbf{h}_{j l}$ in the second set of constraints denotes the channel from BS $n$ to link $l$ (i.e., the index $j$ in the second set of constraints denotes an arbitrary link in $\mathcal{L}(n))$, and the notation $\succeq_{\text {SOC }}$ denotes the generalized inequalities with respect to the second-order cone $[16,19]$.

In problem (8), the objective function and the first set of inequality constraints are separable in $n \in \mathcal{N}$ (one for each BS). Also, it can be easily shown that the second set of inequality constraints of (8) are separable in $n \in \mathcal{N}$. To do this, let us denote $\mathcal{I}_{\text {int }}(n)$ the set of links for which BS $n$ acts as an out-of-cell interferer, i.e., $\mathcal{I}_{\text {int }}(n)=$ $\left\{l \mid l \in \mathcal{L}_{\text {int }}, n \in \mathcal{N}_{\text {int }}(l)\right\}$. Then, by noting that the sets $\left\{(n, l) \mid l \in \mathcal{L}_{\text {int }}, n \in \mathcal{N}_{\text {int }}(l)\right\}$, and $\left\{(n, l) \mid n \in \mathcal{N}, l \in \mathcal{I}_{\text {int }}(n)\right\}$ are identical, the second set of inequality constraints of (8) can be written as

$$
\left[\begin{array}{c}
x_{n, n l} \\
\mathbf{M}_{n}^{\mathrm{H}} \mathbf{h}_{j l}
\end{array}\right] \succeq_{\mathrm{SOC}} 0, \quad n \in \mathcal{N}, l \in \mathcal{I}_{\text {int }}(n),
$$

which is separable in $n \in \mathcal{N}$. Observe that without the consistency constraints, problem (8) can now be easily decoupled into $N$ subproblems, one for each BS.

We next express problem (8) more compactly. To do this, we first express the consistency constraints of problem (8) more compactly by using vector notations, which denote a collection of the local and global variables associated with BS $n$. By using the equivalence between the sets $\left\{(n, l) \mid l \in \mathcal{L}_{\text {int }}, n \in \mathcal{N}_{\text {int }}(l)\right\}$ and $\left\{(n, l) \mid n \in \mathcal{N}, l \in \mathcal{I}_{\text {int }}(n)\right\}$, let us express the consistency constraints of problem (8) as

$$
\begin{aligned}
x_{n, n l} & =z_{n l}, \quad n \in \mathcal{N}, l \in \mathcal{I}_{\text {int }}(n) \\
x_{\operatorname{tran}(l), n l} & =z_{n l}, \quad l \in \mathcal{L}_{\text {int }}, n \in \mathcal{N}_{\text {int }}(l) .
\end{aligned}
$$

In the first set of equalities of (10), $\left\{x_{n, n l}\right\}_{l \in \mathcal{I}_{\text {int }}(n)}$ is a set of local variables that are associated with BS $n$. Similarly, to find a set of local variables that are associated with BS $n$ in the second set of equalities of (10), let us define $\mathcal{L}_{\text {int }}(n)$ the set of links in BS $n$ that are affected by the out-of-cell interference, i.e., $\mathcal{L}_{\text {int }}(n)=\left\{l \mid l \in \mathcal{L}_{\text {int }} \cap \mathcal{L}(n)\right\}$. Then, by noting that the set $\mathcal{L}_{\text {int }}=\cup_{n \in \mathcal{N}} \mathcal{L}_{\text {int }}(n)$, we can rewrite (10) as

$$
\begin{aligned}
x_{n, n l} & =z_{n l}, \quad n \in \mathcal{N}, l \in \mathcal{I}_{\text {int }}(n) \\
x_{\operatorname{tran}(l), b l} & =z_{b l}, \quad n \in \mathcal{N}, l \in \mathcal{L}_{\text {int }}(n), b \in \mathcal{N}_{\text {int }}(l) .
\end{aligned}
$$

Clearly, in the second set of equalities of $(11)^{\mathrm{e}}$, $\left\{x_{\operatorname{tran}(l), b l}\right\}_{l \in \mathcal{L}_{\text {int }}(n), b \in \mathcal{N}_{\text {int }}(l)}$ is a set of local variables that are associated with BS $n$.

We now denote (11) compactly using vector notation. Let us define vectors $\mathbf{x}_{n}$ and $z_{n}$ as ${ }^{\mathrm{f}}$

$$
\begin{aligned}
& \mathbf{x}_{n}=\left\{\left\{x_{n, n l}\right\}_{l \in \mathcal{I}_{\text {int }}(n)},\left\{x_{\operatorname{tran}(l), b l}\right\}_{l \in \mathcal{L}_{\text {int }}(n), b \in \mathcal{N}_{\text {int }}(l)}\right\} \\
& \mathbf{z}_{n}=\left\{\left\{z_{n l}\right\}_{l \in \mathcal{I}_{\text {int }}(n)},\left\{z_{b l}\right\}_{l \in \mathcal{L}_{\text {int }}(n), b \in \mathcal{N}_{\text {int }}(l)}\right\}
\end{aligned}
$$

Then, (11) can be compactly written as

$$
\mathbf{x}_{n}=\mathbf{z}_{n}, \quad n \in \mathcal{N}
$$

Note that $\mathbf{x}_{n}$ is a collection of the local variables that are associated with BS $n$, and $\mathbf{z}_{n}$ is a collection of the global variables that are associate with the components of variable $\mathbf{x}_{n}$. 
Furthermore, for the sake of brevity, let us define the following set

$$
\mathcal{M}_{n}=\left\{\mathbf{M}_{n}, \mathbf{x}_{n}\left|\left[\begin{array}{c}
\sqrt{1+\frac{1}{\gamma_{l}}} \mathbf{h}_{l l}^{\mathrm{H}} \mathbf{m}_{l} \\
\mathbf{M}_{n}^{\mathrm{H}} \mathbf{h}_{l l} \\
\tilde{\mathbf{x}}_{l} \\
\sigma_{l}
\end{array}\right] \succeq_{\operatorname{SOC} 0, \quad l \in \mathcal{L}(n)}\right| \begin{array}{c}
x_{n, n l} \\
\mathbf{M}_{n}^{\mathrm{H}} \mathbf{h}_{j l}
\end{array}\right] \succeq_{\mathrm{SOC}} 0, \quad l \in \mathcal{I}_{\mathrm{int}}(n),
$$

and the following function

$$
f_{n}\left(\mathbf{M}_{n}, \mathbf{x}_{n}\right)= \begin{cases}\sum_{l \in \mathcal{L}(n)}\left\|\mathbf{m}_{l}\right\|_{2}^{2}\left(\mathbf{M}_{n}, \mathbf{x}_{n}\right) \in \mathcal{M}_{n} \\ \infty & \text { otherwise }\end{cases}
$$

Then by using notations (13), (14), and (15), consensus problem (8) can be written compactly as

$$
\begin{array}{cl}
\operatorname{minimize} & \sum_{n \in \mathcal{N}} f_{n}\left(\mathbf{M}_{n}, \mathbf{x}_{n}\right) \\
\text { subject to } & \mathbf{x}_{n}=\mathbf{z}_{n}, \quad n \in \mathcal{N},
\end{array}
$$

where the variables are $\mathbf{M}_{n}, \mathbf{x}_{n}$, and $\mathbf{z}_{n}$ for all $n \in \mathcal{N}$.

\subsection{Distributed algorithm via ADMM: sum power minimization}

In this section, we derive distributed algorithm for problem (16). The proposed algorithm is based on ADMM [21]. We start by writing the augmented Lagrangian [33] for problem (16) as

$$
\begin{aligned}
& L_{\rho}\left(\left\{\mathbf{M}_{n}, \mathbf{x}_{n}\right\}_{n \in \mathcal{N}},\left\{\mathbf{z}_{n}\right\}_{n \in \mathcal{N}},\left\{\mathbf{u}_{n}\right\}_{n \in \mathcal{N}}\right) \\
& \quad=\sum_{n \in \mathcal{N}}\left(f_{n}\left(\mathbf{M}_{n}, \mathbf{x}_{n}\right)+\mathbf{u}_{n}^{T}\left(\mathbf{x}_{n}-\mathbf{z}_{n}\right)+\frac{\rho}{2}\left\|\mathbf{x}_{n}-\mathbf{z}_{n}\right\|_{2}^{2}\right),
\end{aligned}
$$

where $\left\{\mathbf{u}_{n}\right\}_{n \in \mathcal{N}}$ are the dual variables ${ }^{\mathrm{g}}$ associated with the equality constraints of (16), and $\rho>0$ is a penalty parameter that adds the quadratic penalty to the standard Lagrangian $L_{0}$ for the violation of the equality constraints of problem (16).

Each iteration of ADMM algorithm consists of the following three steps [21]

$$
\begin{aligned}
\mathbf{M}_{n}^{i+1}, \mathbf{x}_{n}^{i+1} & =\underset{\mathbf{M}_{n}, \mathbf{x}_{n}}{\operatorname{argmin}} L_{\rho}\left(\mathbf{M}_{n}, \mathbf{x}_{n}, \mathbf{z}_{n}^{i}, \mathbf{u}_{n}^{i}\right), \quad n \in \mathcal{N} \\
\left\{\mathbf{z}_{n}^{i+1}\right\}_{n \in \mathcal{N}} & =\underset{\left\{\mathbf{z}_{n}\right\}_{n \in \mathcal{N}}}{\operatorname{argmin}} L_{\rho}\left(\left\{\mathbf{M}_{n}^{i+1}, \mathbf{x}_{n}^{i+1}\right\}_{n \in \mathcal{N}},\left\{\mathbf{z}_{n}\right\}_{n \in \mathcal{N}},\left\{\mathbf{u}_{n}^{i}\right\}_{n \in \mathcal{N}}\right) \\
\mathbf{u}_{n}^{i+1} & =\mathbf{u}_{n}^{i}+\rho\left(\mathbf{x}_{n}^{i+1}-\mathbf{z}_{n}^{i+1}\right), \quad n \in \mathcal{N},
\end{aligned}
$$

where superscript $i$ is the iteration counter. Steps (18) and (20) are completely decentralized, and hence, they can be carried out independently in parallel in each BS. Note that each component of $\mathbf{z}_{n}$ couples two local variables that are associated with the adjacent BSs (see, consistency constraint of problem $(8))^{\mathrm{h}}$. Thus, step (19) requires to gather the updated local variables $\left(\mathbf{M}_{n}^{i+1}, \mathbf{x}_{n}^{i+1}\right)$ and the dual variables $\mathbf{u}_{n}^{i}$ from all $N$ BSs. In the sequel, we first explain in detail to solve the ADMM steps in (18) and (19). Then, we summarize the proposed ADMM based distributed algorithm.

The local variable update $\left(\mathbf{M}_{n}^{i+1}, \mathbf{x}_{n}^{i+1}\right)$ in (18) is a solution of the following optimization problem

$$
\text { minimize } f_{n}\left(\mathbf{M}_{n}, \mathbf{x}_{n}\right)+\mathbf{u}_{n}^{i T}\left(\mathbf{x}_{n}-\mathbf{z}_{n}^{i}\right)+\frac{\rho}{2}\left\|\mathbf{x}_{n}-\mathbf{z}_{n}^{i}\right\|_{2}^{2}
$$

with variables $\mathbf{M}_{n}$ and $\mathbf{x}_{n}$. Here, we write $\mathbf{u}_{n}^{i T}$ instead of $\left(\mathbf{u}_{n}^{i}\right)^{T}$ to lighten the notation. Let $\mathbf{v}_{n}=(1 / \rho) \mathbf{u}_{n}$ (i.e., $\mathbf{v}_{n}$ is a scaled dual variable). Then by using notations (14) and (15), problem (21) can be equivalently expressed as

$$
\begin{aligned}
\operatorname{minimize} & \sum_{l \in \mathcal{L}(n)}\left\|\mathbf{m}_{l}\right\|_{2}^{2}+\frac{\rho}{2}\left\|\mathbf{x}_{n}-\mathbf{z}_{n}^{i}+\mathbf{v}_{n}^{i}\right\|_{2}^{2} \\
\text { subject to } & {\left[\begin{array}{c}
\sqrt{1+\frac{1}{\gamma_{l}}} \mathbf{h}_{l l}^{\mathrm{H}} \mathbf{m}_{l} \\
\mathbf{M}_{n}^{\mathrm{H}} \mathbf{h}_{l l} \\
\tilde{\mathbf{x}}_{l} \\
\sigma_{l}
\end{array}\right] \succeq_{\text {SOC } 0, \quad l \in \mathcal{L}(n)} } \\
& {\left[\begin{array}{c}
x_{n, n l} \\
\mathbf{M}_{n}^{\mathrm{H}} \mathbf{h}_{j l}
\end{array}\right] \succeq_{\text {SOC } 0, \quad l \in \mathcal{I}_{\text {int }}(n)} }
\end{aligned}
$$

with variables $\mathbf{M}_{n}=\left[\mathbf{m}_{l}\right]_{l \in \mathcal{L}(n)}$ and $\mathbf{x}_{n}$, where $\tilde{\mathbf{x}}_{l}=$ $\left\{x_{n, b l}\right\}_{b \in \mathcal{N}_{\text {int }}(l)}$ is a subset of $\mathbf{x}_{n}$ (see (12)), the matrix $\mathbf{h}_{j l}$ in the second set of constraints denotes the channel from BS $n$ to link $l$ (i.e., the index $j$ in the third set of constraints denotes an arbitrary link in $\mathcal{L}(n)$ ), and the notation $\succeq_{\text {SOC }}$ denotes the generalized inequalities with respect to the second-order cone $[16,19]$. Note that in the objective function of $(22)^{i}$, we have dropped a constant term $\frac{\rho}{2}\left\|\mathbf{v}_{n}^{i}\right\|_{2}^{2}$ since it does not effect the solution of the problem.

Moreover, by writing problem (22) in the epigraph form, and then following the approach of [16, Section IV-B], problem (22) can be equivalently reformulated in the form of second-order cone program (SOCP) as

minimize $t$

$\begin{aligned} \text { subject to } & {\left[\begin{array}{c}t \\ \operatorname{vec}\left(\mathbf{M}_{n}\right) \\ \sqrt{(\rho / 2)}\left(\mathbf{x}_{n}-\mathbf{z}_{n}^{i}+\mathbf{v}_{n}^{i}\right)\end{array}\right] \succeq_{\mathrm{SOC} 0} 0 } \\ & {\left[\begin{array}{c}\sqrt{1+\frac{1}{\gamma_{l}} \mathbf{h}_{l l}^{\mathrm{H}} \mathbf{m}_{l}} \\ \mathbf{M}_{n}^{\mathrm{H}} \mathbf{h}_{l l} \\ \tilde{\mathbf{x}}_{l} \\ \sigma_{l}\end{array}\right] \succeq_{\mathrm{SOC} 0, \quad l \in \mathcal{L}(n)} } \\ & {\left[\begin{array}{c}x_{n, n l} \\ \mathbf{M}_{n}^{\mathrm{H}} \mathbf{h}_{j l}\end{array}\right] \succeq_{\operatorname{SOC} 0, \quad l \in \mathcal{I}_{\mathrm{int}}(n),} }\end{aligned}$ 
with variables $t, \mathbf{M}_{n}$, and $\mathbf{x}_{n}$. Let us denote $t^{\star}, \mathbf{M}_{n}^{\star}$, and $\mathbf{x}_{n}^{\star}$ the solutions of problem (23), then the update $\mathbf{M}_{n}^{i+1}=\mathbf{M}_{n}^{\star}$ and $\mathbf{x}_{n}^{i+1}=\mathbf{x}_{n}^{\star}$.

Now, we turn to the second step of ADMM algorithm and provide a solution for the global variable update (19). The update $\left.\{\mathbf{z}\}_{n}^{i+1}\right\}_{n \in \mathcal{N}}$ is a solution of the following optimization problem

$$
\operatorname{minimize} \sum_{n \in \mathcal{N}}\left(\mathbf{u}_{n}^{i T}\left(\mathbf{x}_{n}^{i+1}-\mathbf{z}_{n}\right)+\frac{\rho}{2}\left\|\mathbf{x}_{n}^{i+1}-\mathbf{z}_{n}\right\|_{2}^{2}\right),
$$

with variable $\left\{\mathbf{z}_{n}\right\}_{n \in \mathcal{N}}$. By using the notations in (12), and further noting equalities (13) and the equality constraints of problem (8) are equivalent, problem (24) in the components of $\mathbf{x}_{n}, \mathbf{z}_{n}$, and $\mathbf{u}_{n}$ can be expressed as

$$
\begin{aligned}
\operatorname{minimize} & \sum_{l \in \mathcal{L}_{\text {int }}} \sum_{n \in \mathcal{N}_{\text {int }}(l)} \sum_{k \in\{n, \operatorname{tran}(l)\}} \\
& \times\left(u_{k, n l}^{i}\left(x_{k, n l}^{i+1}-z_{n l}\right)+\frac{\rho}{2}\left(x_{k, n l}^{i+1}-z_{n l}\right)^{2}\right),
\end{aligned}
$$

with variable $\quad\left\{z_{n l}\right\}_{l \in \mathcal{L}_{\text {int }}, n \in \mathcal{N}_{\text {int }}(l)}$, where $\left\{u_{k, n l}\right\}_{k \in\{n, \operatorname{tran}(l)\}, l \in \mathcal{L}_{\text {int }}, n \in \mathcal{N}_{\text {int }}(l)}$ are the dual variables associated with the equality constraints of problem $(7)^{j}$.

Problem (25) decouples across $z_{n l}$, since the objective function is separable in $z_{n l}$ for all $l \in \mathcal{L}_{\text {int }}, n \in$ $\mathcal{N}_{\text {int }}(l)$. Moreover, the objective function of problem (25) is quadratic in $z_{n l}$. Hence, by setting the gradient of (25) with respect to $z_{n l}$ equal to zero, we can get the solution $z_{n l}^{\star}$ which can be expressed as

$$
z_{n l}^{\star}=\left(x_{n, n l}^{i+1}+x_{\operatorname{tran}(l), n l}^{i+1}+\frac{1}{\rho}\left(u_{n, n l}^{i}+u_{\operatorname{tran}(l), n l}^{i}\right)\right) / 2,
$$

for all $l \in \mathcal{L}_{\text {int }}, n \in \mathcal{N}_{\text {int }}(l)$. Therefore, the update $z_{n l}^{i+1}=$ $z_{n l}^{\star}$ for all $l \in \mathcal{L}_{\text {int }}, n \in \mathcal{N}_{\text {int }}(l)$. Moreover, by substituting $z_{n l}^{i+1}$ in $(20)^{\mathrm{k}}$, we can show that the sum of the dual variables $u_{n, n l}^{i}+u_{\operatorname{tran}(l), n l}^{i}$ is equal to zero. Thus, the update $z_{n l}^{i+1}$ further simplifies to

$$
z_{n l}^{i+1}=\left(x_{n, n l}^{i+1}+x_{\operatorname{tran}(l), n l}^{i+1}\right) / 2,
$$

for all $l \in \mathcal{L}_{\text {int }}, n \in \mathcal{N}_{\text {int }}(l)$. Hence, the global variable update $z_{n l}^{i+1}$ is simply the average of its local copies $x_{n, n l}^{i+1}$ and $x_{\operatorname{tran}(l), n l}^{i+1}$.
Finally, we summarize the proposed ADMM-based distributed algorithm for sum power minimization problem (8) in Algorithm 1.

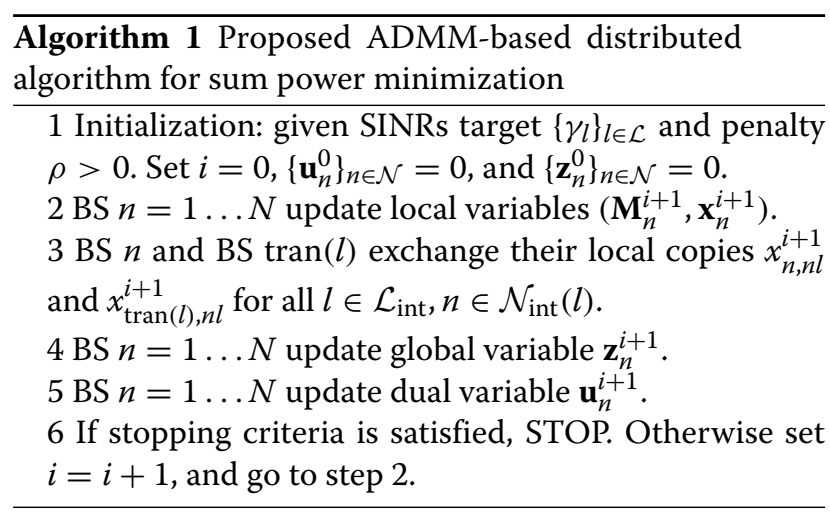

The first step initializes the algorithm. Step 2 updates the local variables of each BS by solving problem (23). Step 2 is completely decentralized. In step 3, the neighboring BSs that are coupled by variable $z_{n l}$, i.e., BS $n$ and BS $\operatorname{tran}(l)$, exchange their local copies $x_{n, n l}^{i+1}$ and $x_{\operatorname{tran}(l), n l}^{i+1}$. In step 4 , each BS update the global variable $\mathbf{z}_{n}^{i+1}$. Note that the global variable update $\mathbf{z}_{n}^{i+1}$ in its component is simply the average of the local copies (27). In step 5, the dual variables are updated by each BS, by solving (20). Step 6 checks the stopping criterial, and the algorithm stops if the stopping criteria is satisfied. Otherwise, the algorithm continues in an iterative manner. A method to find the feasible solution at each iteration of Algorithm 1 is provided in Section 3.3. Note that in deriving Algorithm 1, we have considered perfect CSI in all relevant channels between BSs and receivers. The impact of imperfect CSI in the derivation of the algorithm can be found in [15].

\subsection{Finding feasible solution at each iteration of Algorithm 1}

In many practical applications, we have to stop the distributed algorithm after a finite number of iterations before converging the algorithm. On the other hand, the intermediate solutions provided by Algorithm 1 do not necessarily result feasible solution. In particular, the SINR constraints of problem (4) may not hold since the local copies $x_{n, n l}$ and $x_{\operatorname{tran}(l), n l}$ that correspond to the global variable $z_{n l}$ for all $l \in \mathcal{L}_{\text {int }}, n \in \mathcal{N}_{\text {int }}(l)$ may not be equal. Thus, we can get SINR $\Gamma_{l} \leq \gamma_{l}$ as a solution of step 2 of Algorithm 1 for some $l \in \mathcal{L}$.

At the cost of solving one additional subproblem by each BS in each iteration, we can find a set of feasible beamformers $\mathbf{M}_{n}$ for all $n \in \mathcal{N}$. For this, in order to make the local copies $x_{n, n l}$ and $x_{\operatorname{tran}(l), n l}$ equal, we fix them to the consensus value $z_{n l}^{i}$ (i.e., $x_{n, n l}=z_{n l}^{i}$ and $x_{\operatorname{tran}(l), n l}=z_{n l}^{i}$ ) 
for all $l \in \mathcal{L}_{\text {int }}, n \in \mathcal{N}_{\text {int }}(l)$. Then, solve problem (23) in variables $t$ and $\mathbf{M}_{n}$ by each BS, which can be expressed as

$$
\begin{aligned}
\operatorname{minimize} & t \\
\text { subject to } & {\left[\begin{array}{c}
t \\
\operatorname{vec}\left(\mathbf{M}_{n}\right)
\end{array}\right] \succeq_{\mathrm{SOC}} 0 } \\
& {\left[\begin{array}{c}
\sqrt{1+\frac{1}{\gamma_{l}} \mathbf{h}_{l l}^{\mathrm{H}} \mathbf{m}_{l}} \\
\mathbf{M}_{n}^{\mathrm{H}} \mathbf{h}_{l l} \\
\tilde{\mathbf{x}}_{l} \\
\sigma_{l}
\end{array}\right] \succeq_{\mathrm{SOC} 0, \quad l \in \mathcal{L}(n)} \quad\left[\begin{array}{c}
x_{n, n l}^{i} \\
\mathbf{M}_{n}^{\mathrm{H}} \mathbf{h}_{j l}
\end{array}\right] \succeq_{\mathrm{SOC}} 0, \quad l \in \mathcal{I}_{\text {int }}(n), }
\end{aligned}
$$

where $\tilde{\mathbf{x}}_{l}=\left\{x_{n, b l}\right\}_{b \in \mathcal{N}_{\text {int }}(l)}$. Note that at iteration $i$ the set of beamformer $\left\{\mathbf{M}_{n}\right\}_{n \in \mathcal{N}}$ is feasible for the original problem (4), if problem (28) is feasible for all BSs.

\subsection{Convergence of Algorithm 1 to the global optimum of problem P1}

The convergence of Algorithm 1 to the global optimal solution of problem P1 (i.e., problem (4)) can be established by using proposition [34, Proposition 4.2].

First, by applying proposition [34, Proposition 4.2] to problem (16), we can show that the ADMM Algorithm 1 converges to the global optimal solution of problem (16) (note that problem (16) is compact representation of problem (8)). Next, we note that the phase of the optimization variable $\left\{\mathbf{m}_{l}\right\}_{l \in \mathcal{L}}$ in problems (8) and (4) do not change the objective and the constraints of both problems. Thus, the optimal solution obtained by Algorithm 1 for problem (8) is also optimal for problem (4) (i.e., problem P1).

\section{SINR balancing}

In this section, we derive a distributed algorithm for problem (5), i.e., P2. As before in the sum power minimization problem, we begin by reformulating problem (5) in the global consensus form. Then, we apply ADMM [21] to derive the distributed algorithm.

\subsection{An equivalent reformulation: SINR balancing}

We start by equivalently reformulating SINR balancing problem (5) in the epigraph form [19] as

$$
\begin{array}{ll}
\text { minimize } & -\gamma \\
\text { subject to } & \frac{\left|\mathbf{h}_{l l}^{\mathrm{H}} \mathbf{m}_{l}\right|^{2}}{\sigma_{l}^{2}+\sum_{j \in \mathcal{L}(\operatorname{tran}(l)), j \neq l}\left|\mathbf{h}_{j l}^{\mathrm{H}} \mathbf{m}_{j}\right|^{2}+\sum_{n \in \mathcal{N}_{\text {int }}(l)} z_{n l}^{2}} \geq \gamma, \\
& l \in \mathcal{L} \\
& z_{n l}^{2} \geq \sum_{j \in \mathcal{L}(n)}\left|\mathbf{h}_{j l}^{\mathrm{H}} \mathbf{m}_{j}\right|^{2}, \quad l \in \mathcal{L}_{\text {int }}, n \in \mathcal{N}_{\text {int }}(l) \\
& \sum_{j \in \mathcal{L}(n)}\left\|\mathbf{m}_{l}\right\|_{2}^{2} \leq p_{n}^{\max }, \quad n \in \mathcal{N},
\end{array}
$$

with variables $\gamma,\left\{\mathbf{m}_{l}\right\}_{l \in \mathcal{L}}$, and $\left\{z_{n l}\right\}_{l \in \mathcal{L}_{\text {int }}, n \in \mathcal{N}_{\text {int }}(l)}$.

We now follow a similar approach as in the Section 3.1 to express problem (29) in a global consensus form (i.e., we introduce the local copies of the coupling variables $\gamma$ and $z_{n l}$ for each BS). Since the SINR variable $\gamma$ couples all BSs via a SINR constraints, we introduce local copies $\alpha_{n}$ for each BS such that $\alpha_{n}=\gamma$ for all $n \in \mathcal{N}$. For the outof-cell interference variable $z_{n l}$, we introduce local copies $x_{k, n l}$ and $x_{\operatorname{tran}(l), n l}$, respectively, for BS $n$ and BS $\operatorname{tran}(l)$ as in problem (7). Then, problem (29) in the global consensus form can be expressed equivalently as

$$
\begin{aligned}
& \text { minimize }-\gamma \\
& \text { subject to } \frac{\left|\mathbf{h}_{l l}^{\mathrm{H}} \mathbf{m}_{l}\right|^{2}}{\sigma_{l}^{2}+\sum_{j \in \mathcal{L}(n), j \neq l}\left|\mathbf{h}_{j l}^{\mathrm{H}} \mathbf{m}_{j}\right|^{2}+\sum_{b \in \mathcal{N}_{\text {int }}(l)} x_{n, b l}^{2}} \geq \alpha_{n}, \\
& n \in \mathcal{N}, l \in \mathcal{L}(n) \\
& x_{n, n l}^{2} \geq \sum_{j \in \mathcal{L}(n)}\left|\mathbf{h}_{j l}^{\mathrm{H}} \mathbf{m}_{j}\right|^{2}, \quad n \in \mathcal{N}, l \in \mathcal{I}_{\text {int }}(n) \\
& \sum_{j \in \mathcal{L}(n)}\left\|\mathbf{m}_{l}\right\|_{2}^{2} \leq p_{n}^{\max }, \quad n \in \mathcal{N} \\
& x_{k, n l}=z_{n l}, \quad k \in\{n, \operatorname{tran}(l)\}, l \in \mathcal{L}_{\text {int }}, n \in \mathcal{N}_{\text {int }}(l) \\
& \alpha_{n}=\gamma, \quad n \in \mathcal{N} \text {, }
\end{aligned}
$$

with variables $\quad \gamma, \quad\left\{\mathbf{m}_{l}\right\}_{l \in \mathcal{L}}, \quad\left\{\alpha_{n}\right\}_{n \in \mathcal{N}}$, $\left\{x_{k, n l}\right\}_{k \in\{n, \operatorname{tran}(l)\}, n \in \mathcal{N}, l \in \mathcal{I}_{\text {int }}(n)}$, and $\left\{z_{n l}\right\}_{l \in \mathcal{L}_{\text {int }}, n \in \mathcal{N}_{\text {int }}(l)}$. Note that in the second set of inequality constraints, we use the equivalence between the sets $\left\{(n, l) \mid l \in \mathcal{L}_{\text {int }}, n \in \mathcal{N}_{\text {int }}(l)\right\}$ and $\left\{(n, l) \mid n \in \mathcal{N}, l \in \mathcal{I}_{\text {int }}(n)\right\}$ (see (10)).

Now, we express problem (30) more compactly. Note that except the third inequality constraints and the last equality constraints of problem (30), the constraint set of problem (30) is identical to that of problem (7). Hence, we can use variables $\mathbf{M}_{n}, \mathbf{x}_{n}$, and $\mathbf{z}_{n}$ to define the set $C_{n}$ as given in (31)

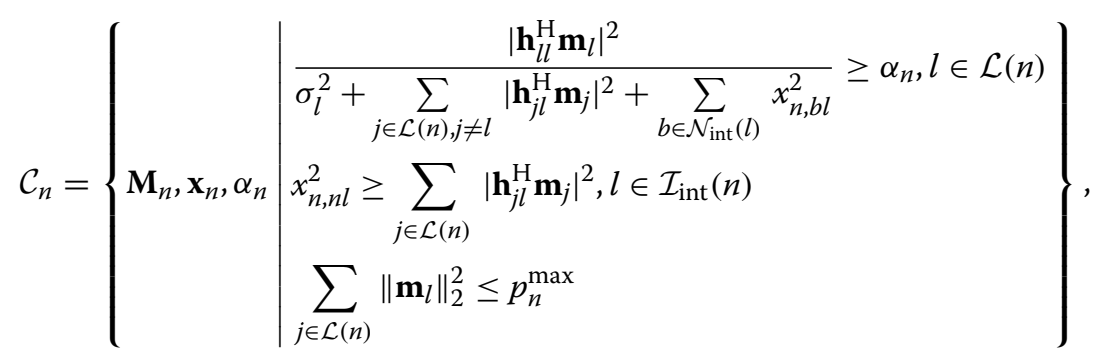


and the following indicator function $I_{n}\left(\mathbf{M}_{n}, \mathbf{x}_{n}, \alpha_{n}\right)$

$$
I_{n}\left(\mathbf{M}_{n}, \mathbf{x}_{n}, \alpha_{n}\right)= \begin{cases}0 & \left(\mathbf{M}_{n}, \mathbf{x}_{n}, \alpha_{n}\right) \in \mathcal{C}_{n} \\ \infty & \text { otherwise }\end{cases}
$$

Then, by using notations (31) and (32), consensus problem (30) can be rewritten compactly as

$$
\begin{gathered}
\operatorname{minimize}-\gamma+\sum_{n \in \mathcal{N}} I_{n}\left(\mathbf{M}_{n}, \mathbf{x}_{n}, \alpha_{n}\right) \\
\text { subject to } \mathbf{x}_{n}=\mathbf{z}_{n}, \quad n \in \mathcal{N} \\
\alpha_{n}=\gamma, \quad n \in \mathcal{N},
\end{gathered}
$$

with variables $\gamma$ and $\left\{\mathbf{M}_{n}, \mathbf{x}_{n}, \mathbf{z}_{n}, \alpha_{n}\right\}_{n \in \mathcal{N}}$. Furthermore, by noting that $\sum_{n \in \mathcal{N}} \alpha_{n}=N \gamma$ (from the second equality constraints of (33)), problem (33) can be expressed equivalently as

$$
\begin{gathered}
\operatorname{minimize} \sum_{n \in \mathcal{N}}\left(-\frac{\alpha_{n}}{N}+I_{n}\left(\mathbf{M}_{n}, \mathbf{x}_{n}, \alpha_{n}\right)\right) \\
\text { subject to } \mathbf{x}_{n}=\mathbf{z}_{n}, \quad n \in \mathcal{N} \\
\alpha_{n}=\gamma, \quad n \in \mathcal{N},
\end{gathered}
$$

with variables $\gamma$ and $\left\{\mathbf{M}_{n}, \mathbf{x}_{n}, \mathbf{z}_{n}, \alpha_{n}\right\}_{n \in \mathcal{N}}$.

\subsection{Distributed algorithm via ADMM: SINR balancing}

To derive the ADMM algorithm, we first form the augmented Lagrangian [33] of problem (34). Let $\mathbf{u}_{n}$ and $v_{n}$ be the dual variables associated with the first and second consensus constraints of problem (34), respectively. Then, the augmented Lagrangian can be written as

$$
\begin{aligned}
& L_{\rho}\left(\left\{\mathbf{M}_{n}, \mathbf{x}_{n}, \alpha_{n}, \mathbf{u}_{n}, v_{n}, \mathbf{z}_{n}\right\}_{n \in \mathcal{N}}, \gamma\right) \\
& =\sum_{n \in \mathcal{N}}\left(-\frac{\alpha_{n}}{N}+I_{n}\left(\mathbf{M}_{n}, \mathbf{x}_{n}, \alpha_{n}\right)+\mathbf{u}_{n}^{T}\left(\mathbf{x}_{n}-\mathbf{z}_{n}\right)\right. \\
& \left.\quad+v_{n}\left(\alpha_{n}-\gamma\right)+\frac{\rho}{2}\left\|\mathbf{x}_{n}-\mathbf{z}_{n}\right\|_{2}^{2}+\frac{\rho}{2}\left(\alpha_{n}-\gamma\right)^{2}\right),
\end{aligned}
$$

where $\rho>0$ is the penalty parameter. Each iteration of ADMM consists of the following steps [21]

$$
\begin{aligned}
& \mathbf{M}_{n}^{i+1}, \mathbf{x}_{n}^{i+1}, \alpha_{n}^{i+1}= \underset{\mathbf{M}_{n}, \mathbf{x}_{n}, \alpha_{n}}{\operatorname{argmin}} L_{\rho}\left(\mathbf{M}_{n}, \mathbf{x}_{n}, \alpha_{n}, \mathbf{u}_{n}^{i}, v_{n}^{i}, \mathbf{z}_{n}^{i}, \gamma^{i}\right) \\
& n \in \mathcal{N} \\
&\left\{\mathbf{z}_{n}^{i+1}\right\}_{n \in \mathcal{N}}, \gamma^{i+1}= \underset{\left\{\mathbf{z}_{n}\right\}_{n \in \mathcal{N}}, \gamma}{\operatorname{argmin}} L_{\rho}\left(\left\{\mathbf{M}_{n}^{i+1}, \mathbf{x}_{n}^{i+1}, \alpha_{n}^{i+1}, \mathbf{u}_{n}^{i}\right.\right. \\
&\left.\left.v_{n}^{i}, \mathbf{z}_{n}\right\}_{n \in \mathcal{N}}, \gamma\right) \\
& \mathbf{u}_{n}^{i+1}= \mathbf{u}_{n}^{i}+\rho\left(\mathbf{x}_{n}^{i+1}-\mathbf{z}_{n}^{i+1}\right), \quad n \in \mathcal{N} \\
& v_{n}^{i+1}= v_{n}^{i}+\rho\left(\alpha_{n}^{i+1}-\gamma^{i+1}\right), \quad n \in \mathcal{N}
\end{aligned}
$$

Note that the first step is completely decentralized. Each BS $n \in \mathcal{N}$ updates the local variables $\left(\mathbf{M}_{n}^{i+1}, \mathbf{x}_{n}^{i+1}, \alpha_{n}^{i+1}\right)$ by solving the following optimization problem

$$
\begin{aligned}
\operatorname{minimize} & -\frac{\alpha_{n}}{N}+I_{n}\left(\mathbf{M}_{n}, \mathbf{x}_{n}, \alpha_{n}\right)+\mathbf{u}_{n}^{i T}\left(\mathbf{x}_{n}-\mathbf{z}_{n}^{i}\right) \\
& +v_{n}^{i}\left(\alpha_{n}-\gamma^{i}\right)+\frac{\rho}{2}\left\|\mathbf{x}_{n}-\mathbf{z}_{n}^{i}\right\|_{2}^{2}+\frac{\rho}{2}\left(\alpha_{n}-\gamma^{i}\right)^{2},
\end{aligned}
$$

with variables $\alpha_{n}, \mathbf{M}_{n}$, and $\mathbf{x}_{n}$. Let $\mathbf{v}_{n}=(1 / \rho) \mathbf{u}_{n}$ and $\lambda_{n}=$ $(1 / \rho) v_{n}$, then by combining the linear and quadratic terms of the objective function ${ }^{\mathrm{m}}$, problem (40) can be written as

$$
\begin{aligned}
\operatorname{minimize} & -\frac{\alpha_{n}}{N}+I_{n}\left(\mathbf{M}_{n}, \mathbf{x}_{n}, \alpha_{n}\right)+\frac{\rho}{2}\left\|\mathbf{x}_{n}-\mathbf{z}_{n}^{i}+\mathbf{v}_{n}^{i}\right\|_{2}^{2} \\
& +\frac{\rho}{2}\left(\alpha_{n}-\gamma^{i}+\lambda_{n}^{i}\right)^{2},
\end{aligned}
$$

with variables $\alpha_{n}, \mathbf{M}_{n}$, and $\mathbf{x}_{n}$. Note that in the objective function of (41), constant terms $\frac{\rho}{2}\left\|\mathbf{v}_{n}^{i}\right\|_{2}^{2}$ and $\frac{\rho}{2}\left(\lambda_{n}^{i}\right)^{2}$ are dropped, since they do not affect the solution of the optimization problem.

Problem (41) is not a convex problem, due to the indicator function $I_{n}\left(\mathbf{M}_{n}, \mathbf{x}_{n}, \alpha_{n}\right)$ is a function of nonconvex set $\mathcal{C}_{n}$ (see (31)). However, for fixed $\alpha_{n}$, set $\mathcal{C}_{n}$ is a convex set, and hence, problem (41) can be solved easily. Therefore, to solve problem (41), we first find the optimal $\alpha_{n}^{\star}$ and then find $\mathbf{M}_{n}^{\star}$ and $\mathbf{x}_{n}^{\star}$.

For fixed $\alpha_{n}$, let us denote the optimal value function of problem (41) as

$$
\begin{aligned}
p\left(\alpha_{n}\right)= & \inf _{\mathbf{M}_{n}, \mathbf{x}_{n}}\left(-\frac{\alpha_{n}}{N}+I_{n}\left(\mathbf{M}_{n}, \mathbf{x}_{n}, \alpha_{n}\right)+\frac{\rho}{2}\left\|\mathbf{x}_{n}-\mathbf{z}_{n}^{i}+\mathbf{v}_{n}^{i}\right\|_{2}^{2}\right. \\
& \left.+\frac{\rho}{2}\left(\alpha_{n}-\gamma^{i}+\lambda_{n}^{i}\right)^{2}\right) \\
= & \inf _{\mathbf{M}_{n}, \mathbf{x}_{n}}\left(I_{n}\left(\mathbf{M}_{n}, \mathbf{x}_{n}, \alpha_{n}\right)+\frac{\rho}{2}\left\|\mathbf{x}_{n}-\mathbf{z}_{n}^{i}+\mathbf{v}_{n}^{i}\right\|_{2}^{2}\right) \\
& -\frac{\alpha_{n}}{N}+\frac{\rho}{2}\left(\alpha_{n}-\gamma^{i}+\lambda_{n}^{i}\right)^{2},
\end{aligned}
$$

where (43) follows by noting that $\alpha_{n} / N$ and $\frac{\rho}{2}\left(\alpha_{n}-\gamma^{i}+\right.$ $\left.\lambda_{n}^{i}\right)^{2}$ are independent of the optimization variables $\mathbf{M}_{n}$ and $\mathbf{x}_{n}$. Then, the optimal value of problem (41) is given by

$$
p^{\star}=\inf _{\alpha_{n}} p\left(\alpha_{n}\right)
$$

For ease of presentation, let us express the optimal value function $p\left(\alpha_{n}\right)$ in (43) as

$$
p\left(\alpha_{n}\right)=\tilde{p}\left(\alpha_{n}\right)-\frac{\alpha_{n}}{N}+\frac{\rho}{2}\left(\alpha_{n}-\gamma^{i}+\lambda_{n}^{i}\right)^{2},
$$

where $\tilde{p}\left(\alpha_{n}\right)$ is the optimal value of the following optimization problem 


$$
\begin{array}{ll}
\text { minimize } & \frac{\rho}{2}\left\|\mathbf{x}_{n}-\mathbf{z}_{n}^{i}+\mathbf{v}_{n}^{i}\right\|_{2}^{2} \\
\text { subject to } & \frac{\left|\mathbf{h}_{l l}^{\mathrm{H}} \mathbf{m}_{l}\right|^{2}}{\sigma_{l}^{2}+\sum_{j \in \mathcal{L}(n), j \neq l}\left|\mathbf{h}_{j l}^{\mathrm{H}} \mathbf{m}_{j}\right|^{2}+\sum_{b \in \mathcal{N}_{\text {int }}(l)} x_{n, b l}^{2}} \geq \alpha_{n}, \\
& l \in \mathcal{L}(n) \\
& x_{n, n l}^{2} \geq \sum_{j \in \mathcal{L}(n)}\left|\mathbf{h}_{j l}^{\mathrm{H}} \mathbf{m}_{j}\right|^{2}, \quad l \in \mathcal{I}_{\text {int }}(n) \\
& \sum_{j \in \mathcal{L}(n)}\left\|\mathbf{m}_{l}\right\|_{2}^{2} \leq p_{n}^{\max },
\end{array}
$$

with variables $\mathbf{x}_{n}$ and $\left\{\mathbf{m}_{l}\right\}_{l \in \mathcal{L}(n)}$. Note that to write (46), we have used the notations defined in (31) and (32).

Let the interval $\left[0, \alpha_{n}^{\max }\right]$ denote the range of feasible $\alpha_{n}$ for problem (46). Note that the optimal value $\tilde{p}\left(\alpha_{n}\right)$ is a nondecreasing function of $\alpha_{n} \in\left[0, \alpha_{n}^{\max }\right]$ (see Appendix 1). Based on this observation, in Appendix 1, we have provided the condition for which $p\left(\alpha_{n}\right)$ is a unimodal function and propose the bracketing method [30,31] to solve problem (44). In Algorithm 2, we summarize the bracketing method (golden ratio search) [30, Section 8.1] to find the optimal $\alpha_{n}^{\star}$ for problem (44).

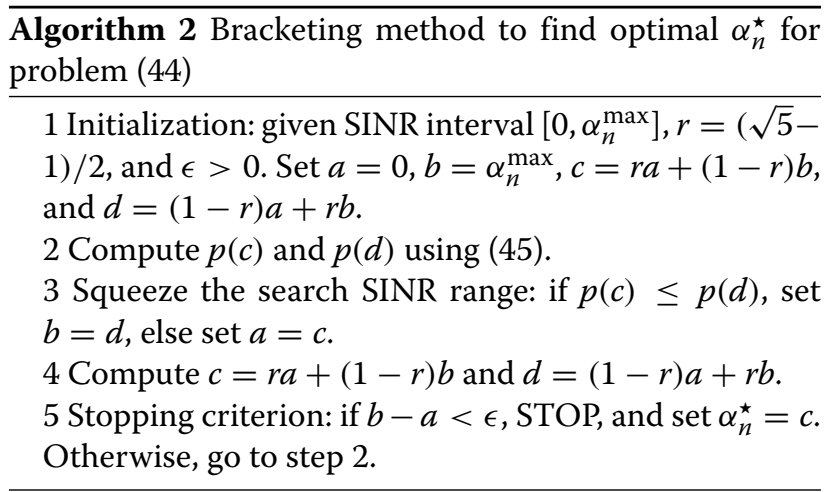

Next, we find $\mathbf{x}_{n}^{\star}$ and $\mathbf{M}_{n}^{\star}=\left\{\mathbf{m}_{l}^{\star}\right\}_{l \in \mathcal{L}(n)}$ that are associated with $\alpha_{n}^{\star}$ by solving problem (46). By writing problem (46) in the epigraph form, and then following the approach of [16, Section IV-B], problem (46) can be formulated equivalently in the form of SOCP as

$$
\begin{aligned}
\operatorname{minimize} & t \\
\text { subject to } & {\left[\begin{array}{c}
t \\
\sqrt{\rho / 2}\left(\mathbf{x}_{n}-\mathbf{z}_{n}^{i}+\mathbf{v}_{n}^{i}\right)
\end{array}\right] \succeq_{\text {SOC } 0} } \\
& {\left[\begin{array}{c}
\sqrt{1+\frac{1}{\alpha_{n}}} \mathbf{h}_{l l}^{\mathrm{H}} \mathbf{m}_{l} \\
\mathbf{M}_{n}^{\mathrm{H}} \mathbf{h}_{l l} \\
\tilde{\mathbf{x}}_{l} \\
\sigma_{n}
\end{array}\right] \succeq_{\text {SOC } 0, \quad l \in \mathcal{L}(n)} } \\
& {\left[\begin{array}{c}
x_{n, n l} \\
\mathbf{M}_{n}^{\mathrm{H}} \mathbf{h}_{j l}
\end{array}\right] \succeq_{\text {SOC } 0, \quad l \in \mathcal{I}_{\text {int }}(n)} } \\
& {\left[\begin{array}{c}
\sqrt{p_{n}^{\max }} \\
\operatorname{vec}\left(\mathbf{M}_{n}\right)
\end{array}\right] \succeq_{\text {SOC } 0,} }
\end{aligned}
$$

with variables $t, \mathbf{x}_{n}$, and $\mathbf{M}_{n}$, where $\tilde{\mathbf{x}}_{l}=\left\{x_{n, b l}\right\}_{b \in \mathcal{N}_{\text {int }}(l)}$ is a subset of $\mathbf{x}_{n}$ (see (12)), the matrix $\mathbf{h}_{j l}$ in the third set of constraints denotes the channel from BS $n$ to link $l$ (i.e., the index $j$ in the third set of constraints denotes an arbitrary link in $\mathcal{L}(n))$. Note that to write problem (46) in the SOCP form (47), we first took the square root of the objective function of (46). Hence, the optimal value of problem (46) is given by $t^{\star 2}$ (i.e., $\tilde{p}\left(\alpha_{n}^{\star}\right)=t^{\star 2}$ ), where $t^{\star}$ is the solution of problem (47).

We now turn to the second step of ADMM in (37), where the global variables $\left\{\mathbf{z}_{n}\right\}_{n \in \mathcal{N}}^{i+1}$ and $\gamma^{i+1}$ are updated. By dropping the constant terms which do not affect the solution, problem (37) can be written as

$$
\begin{aligned}
\operatorname{minimize} & \sum_{n \in \mathcal{N}}\left(\mathbf{u}_{n}^{i T}\left(\mathbf{x}_{n}^{i+1}-\mathbf{z}_{n}\right)+v_{n}^{i}\left(\alpha_{n}^{i+1}-\gamma\right)\right. \\
& \left.+\frac{\rho}{2}\left\|\mathbf{x}_{n}^{i+1}-\mathbf{z}_{n}\right\|_{2}^{2}+\frac{\rho}{2}\left(\alpha_{n}^{i+1}-\gamma\right)^{2}\right),
\end{aligned}
$$

with variables $\left\{\mathbf{z}_{n}\right\}_{n \in \mathcal{N}}$ and $\gamma$.

Problem (48) is separable in variables $\left\{\mathbf{z}_{n}\right\}_{n \in \mathcal{N}}$ and $\gamma$. Note that minimization of problem (48) with respect to $\left\{\mathbf{z}_{n}\right\}_{n \in \mathcal{N}}$ yields problem (24), and hence, the solution $\left\{\mathbf{z}_{n}^{\star}\right\}_{n \in \mathcal{N}}$ is given by (27). Here, we provide the solution for $\gamma$. Minimization of problem (48) with respect to $\gamma$ yields the following optimization problem

$$
\operatorname{minimize} \sum_{n \in \mathcal{N}}\left(v_{n}^{i}\left(\alpha_{n}^{i+1}-\gamma\right)+\frac{\rho}{2}\left(\alpha_{n}^{i+1}-\gamma\right)^{2}\right) .
$$

Problem (49) is an unconstrained quadratic optimization problem in $\gamma$. Therefore, by setting the gradient of problem (49) with respect to $\gamma$ equal to zero, we can get

$$
\gamma^{\star}=\frac{\sum_{n \in \mathcal{N}} v_{n}^{i}+\rho \alpha_{n}^{i+1}}{\rho N} .
$$

Hence, the update $\gamma^{i+1}=\gamma^{\star}$. Moreover, by substituting $\gamma^{i+1}$ in (39), we can show that the sum of the dual variables $\sum_{n \in \mathcal{N}} v_{n}^{i}$ is equal to zero. Thus, the update $\gamma^{i+1}$ (i.e., (50)) further simplifies to

$$
\gamma^{\star}=\frac{\sum_{n \in \mathcal{N}} \alpha_{n}^{i+1}}{N}
$$

We now summarize the proposed ADMM-based distributed algorithm for SINR balancing problem in Algorithm 3. 


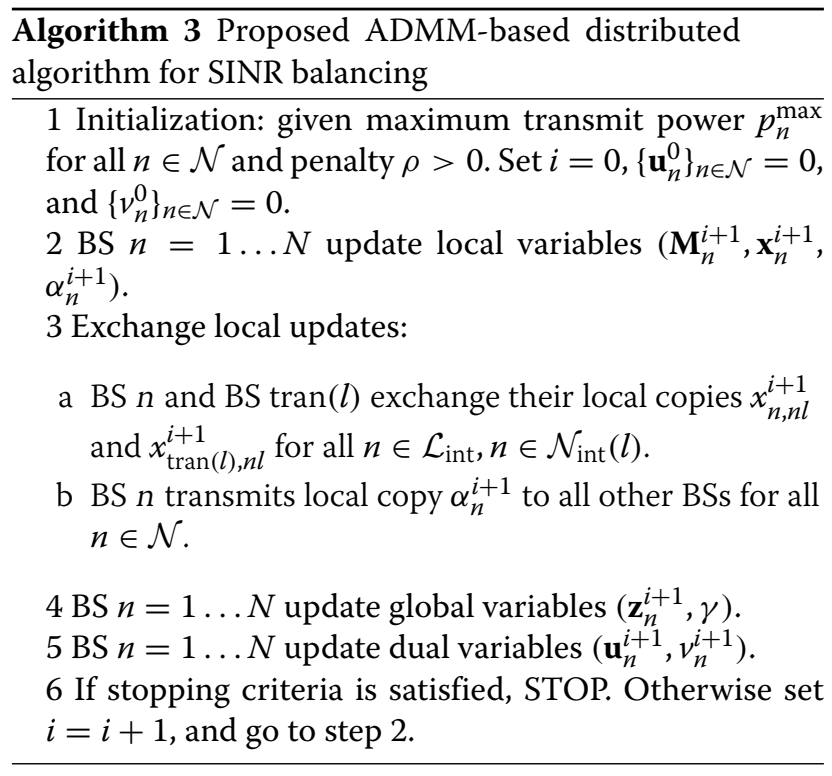

The computational steps of Algorithm 3 is similar to that of Algorithm 1. As in Algorithm 1, step 1 initializes the algorithm. Step 2 updates the local variables. In step 3, BSs exchange their local copies to update the global variables. Local copies $x_{n, n l}^{i+1}$ and $x_{\operatorname{tran}(l), n l}^{i+1}$ are exchanged between the adjacent BS $n$ and BS tran $(l)$, while local copy $\alpha_{n}$ is broadcasted to all other BSs. Steps 4 and 5 updates the global and dual variables, respectively. Note that steps 2, 4, and 5 are completely decentralized. Step 6 checks the stopping criterian ${ }^{\mathrm{n}}$. A method to find the feasible solution at each iteration of Algorithm 3 is provided in next section.

\subsection{Finding feasible solution at each iteration of Algorithm 3}

Note that at each step of Algorithm 3, the locally obtained SINR $\alpha_{n}$ for all $n \in \mathcal{N}$ are not necessarily balanced (i.e., $\alpha_{n}$ for all $n \in \mathcal{N}$ are not necessarily equal). So, we can take the global variable $\gamma^{i}$, which is the average of $\alpha_{n}$ for all $n \in$ $\mathcal{N}$, as the intermediate solution of Algorithm 3. However, due to the difference in the local copies $x_{n, n l}^{i+1}$ at BS $n$ and $x_{\operatorname{tran}(l), n l}^{i+1}$ at BS $\operatorname{tran}(l)$, and the maximum transmit power constraint of the BSs, the intermediate solution $\gamma^{i}$ may not be feasible for all BSs.

Therefore, it is necessary to check the feasibility of $\gamma^{i}$ to use it as the intermediate solution at each step of Algorithm 3. The SINR $\gamma^{i}$ is feasible for BS $n$, if their exist a feasible solution of problem (47) for $\alpha_{n}=\gamma^{i}$ and given out-of-cell interference value $\mathbf{x}_{n}$. Thus, we set $\alpha_{n}=\gamma^{i}$ and $\mathbf{x}_{n}=\mathbf{z}_{n}^{i}$ for all $n \in \mathcal{N}$ (i.e., $\alpha_{n}$ and $\mathbf{x}_{n}$ are set equal to the consensus value). Then, check the feasibility of problem (47) by each BS in between steps 4 and 5 of Algorithm 3, which is equivalent to the following SOCP feasibility problem

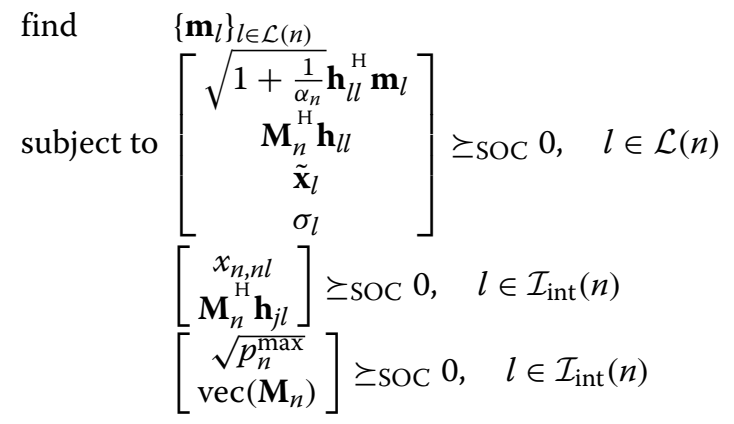

with variable $\mathbf{M}_{n}=\left[\mathbf{m}_{l}\right]_{l \in \mathcal{L}(n)}$, where $\tilde{\mathbf{x}}_{l}=\left\{x_{n, b l}\right\}_{b \in \mathcal{N}_{\text {int }}(l)}$ is a subset of $\mathbf{x}_{n}$ (see (12)), the matrix $\mathbf{h}_{j l}$ in the third set of constraints denotes the channel from BS $n$ to link $l$ (i.e., the index $j$ in the third set of constraints denotes an arbitrary link in $\mathcal{L}(n))$. Note that $\gamma^{i}$ is feasible for the original problem (5) only if problem (52) is feasible for all BSs. Thus, in Algorithm 3, we can update the feasible SINR $\gamma_{\text {feas }}^{i}$ as

$\gamma_{\text {feas }}^{i}= \begin{cases}\gamma^{i} & \text { if problem (52) is feasiblem for all } n \in \mathcal{N} \\ \gamma_{\text {feas }}^{i-1} & \text { otherwise, }\end{cases}$

where $\gamma_{\text {feas }}^{0}=0$.

\section{Numerical example}

In this section, we numerically evaluate the performance of proposed Algorithms 1 and 3. In our simulations, two multicell wireless networks as shown in Figure 1 are considered. In the case of first network (i.e., Figure 1a), there are $N=2$ BSs with $T=4$ antennas at each one. The distance between the BSs is denoted by $D_{\mathrm{BS}}$. In the case of second network (i.e., Figure $1 \mathrm{~b}$ ), there are $N=7$ BSs with $T=6$ antennas at each one. The BSs are located such that they form the hexagon, and the distance between the BSs is denoted by $D_{\mathrm{BS}}$. We assume that BSs have circular transmission and interference regions, where the radius of the transmission region of each $\mathrm{BS}$ is denoted by $R_{\mathrm{BS}}$, and the radius of the interference region of each BS is denoted by $R_{\text {int }}$. For simplicity, we assume 4 users per cell in the first network, and three users per cell in the second network. The location of users associated with BSs is arbitrarily chosen as shown in Figure 1.

We assume an exponential path loss model, where the channel matrix between BSs and users is modeled as

$$
\mathbf{h}_{j l}=\left(\frac{d_{j l}}{d_{0}}\right)^{-\eta / 2} \mathbf{c}_{j l}
$$

where $d_{j l}$ is the distance from the transmitter of data stream $j$ (i.e., BS $\operatorname{tran}(j))$ to the receiver of data stream $l$ (i.e., user rec $(l)), d_{0}$ is the far field reference distance [35], 
$\eta$ is the path loss exponent, and $\mathbf{c}_{j l} \in C^{T}$ is arbitrarily chosen from the distribution $\mathcal{C N}(0, \mathbf{I})$ (i.e., frequencyflat fading channel with uncorrelated antennas). Here, we refer an arbitrarily generated set of fading coefficients $\mathcal{C}=$ $\left\{\mathbf{c}_{j l} \mid j, l \in \mathcal{L}\right\}$ as a single fading realization.

We assume that the maximum power constraint is same for each BS, i.e., $p_{n}^{\max }=p_{0}^{\max }$ for all $n \in \mathcal{N}$, and $\sigma_{l}=\sigma$ for all $l \in \mathcal{L}$. We define the signal-to-noise ratio (SNR) operating point at a distance $r$ as

$$
\operatorname{SNR}(r)=\left(\frac{r}{d_{0}}\right)^{-\eta} \frac{p_{0}^{\max }}{\sigma^{2}}
$$

In our simulations, we set $d_{0}=1, \eta=4, \sigma^{2}=1$, $p_{0}^{\max } / \sigma^{2}=45 \mathrm{~dB}, \operatorname{SNR}\left(R_{\text {int }}\right)=0 \mathrm{~dB}, \operatorname{SNR}\left(R_{\mathrm{BS}}\right)=5 \mathrm{~dB}$, and $D_{\mathrm{BS}}=1.5 \times R_{\mathrm{BS}}$.

To illustrate the convergence behavior of Algorithm 1, we consider a single fading realization and run the algorithm for both networks shown in Figure 1. For a comparison, we consider a dual decomposition-based distributed algorithm (DDA) proposed in [12]. For DDA [12], we consider fixed step size $\alpha$ to solve the master problem (see in [12]), which is based on the subgradient method [18].

Figure 3 shows the normalized power accuracy $\mid p^{i}-$ $p^{\star} \mid / p^{\star}$, where $p^{i}$ is the objective value at $i$ th iteration, and $p^{\star}$ is the optimal objective value obtained by using centralized algorithm [16, Section IV]. SINR target is set to $\gamma_{l}=5 \mathrm{~dB}$ for all $l \in \mathcal{L}$. DDA [12] plots are drawn for the subgradient step size $\alpha=10,50,100$. For Algorithm 1, the penalty parameter is set to $\rho=$ $0.5 \beta, \beta, 2 \beta$, where $\beta$ depends on the problem parameters (detailed in Appendix 2) and it is defined as

$$
\beta=\max _{n \in \mathcal{N}}\left\{\sum_{l \in \mathcal{L}(n)}\left(10^{0.1 \times \gamma_{l}}\right) /\left\|\mathbf{h}_{l l}\right\|_{2}^{2}\right\} .
$$

Results show that the proposed Algorithm 1 converges much faster than DDA [12]. For example, in both multicell networks, Algorithm 1 can achieve normalized power accuracy $10^{-2}$ in less than 10 iterations. However, in order to gain the same accuracy (i.e., normalized power accuracy $10^{-2}$ ), DDA [12] requires more than 200 iterations for all simulated cases in both networks. Results also show that Algorithm 1 performs very well for a wide range of values of $\rho$. Hence, Algorithm 1 is less sensitive to the variation of values of $\rho$, while the results show that the convergence speed of DDA [12] is quite sensitive to the variation of the subgradient step size $\alpha$.

In order to see the average behavior of the proposed Algorithm 1, we next consider fading case. Here, we run Algorithm 1 for 500 fading realizations with the algorithm parameter $\rho=2 \beta$ for both networks shown in Figure 1 . We first present the feasibility rate of the proposed algorithm, and then, we provide the average performance of the algorithm.

Figure 4 shows the feasibility rate of Algorithm 1 versus iteration for SINR target $\gamma_{l}=5 \mathrm{~dB}$ and $15 \mathrm{~dB}$ for all $l \in \mathcal{L}$. For a comparison, we consider DDA [12] with the subgradient step size $\alpha=50$. Plots are drawn for the first 50 iterations. Results show that the proposed algorithm can achieves the feasible solution for all channel realizations (for multicell network 1a, the proposed algorithm achieves the feasible solution for all simulated cases; and for multicell network $1 \mathrm{~b}$, the feasibility rate improves with the iteration). However, for DDA [12] feasibility rate depends on the network size and the SINR target. For

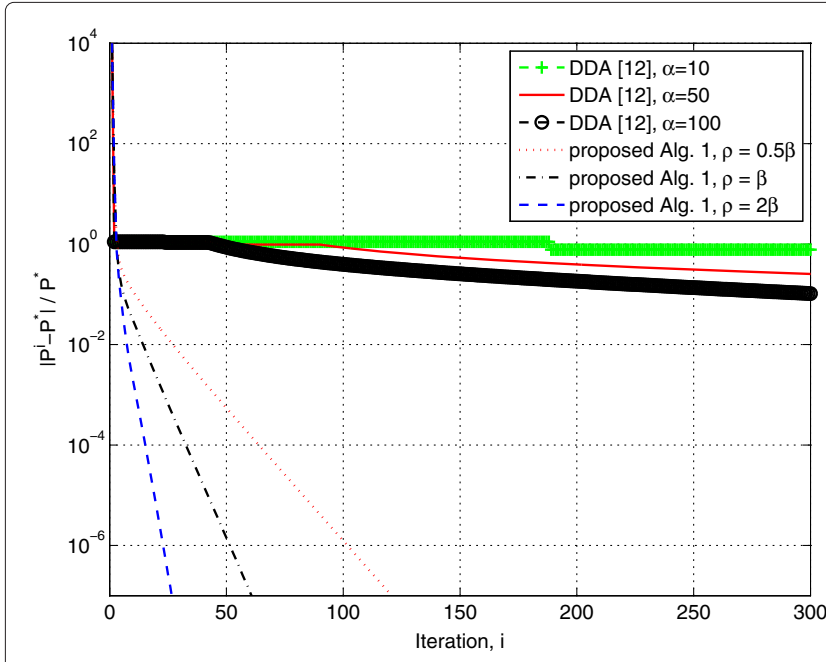

(a)

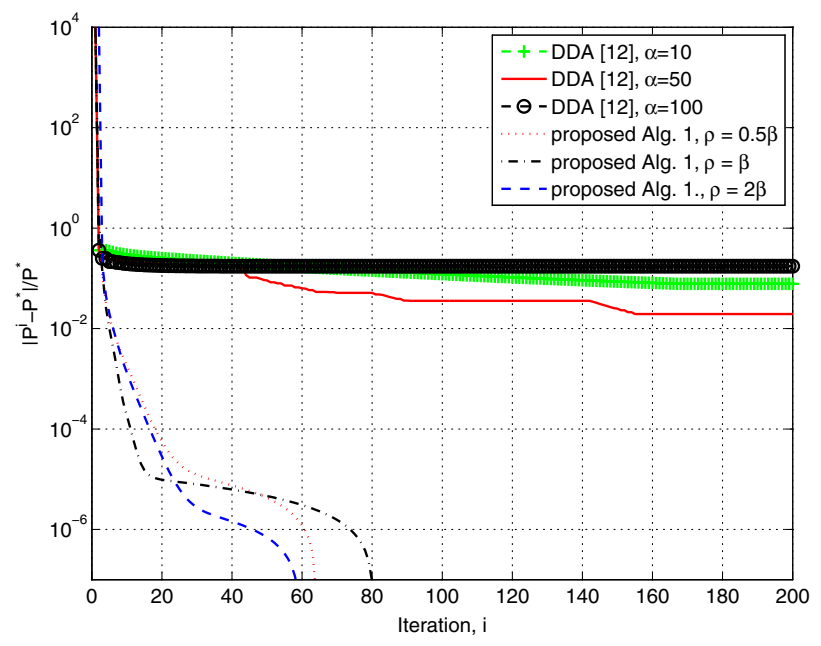

(b)

Figure 3 Normalized accuracy versus iteration. Normalized power accuracy versus iteration for SINR $\gamma_{\mid}=5 \mathrm{~dB}$ for all / $\in \mathcal{L}$ : (a) Multicell network 1; (b) Multicell network 2. 


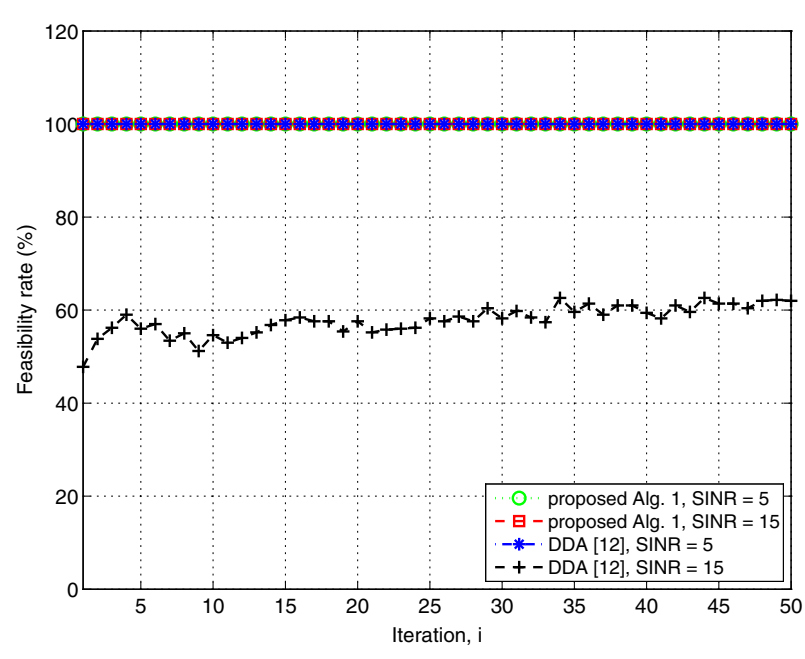

(a)

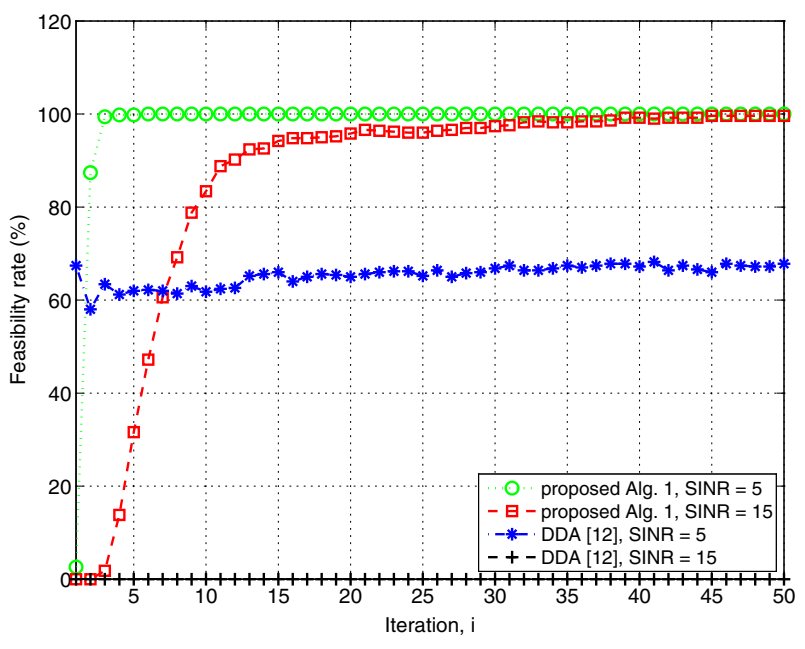

(b)

Figure 4 Feasibility rate versus iteration. Feasibility rate versus iteration for SINR target $\gamma_{l}=5 \mathrm{~dB}$ and $15 \mathrm{~dB}$ for all / $\in \mathcal{L}$ : (a) Multicell network 1; (b) Multicell network 2.

example, in the case of small network and low SINR target (i.e., multicell network $1 \mathrm{a}$ and SINR target $\gamma_{l}=5 \mathrm{~dB}$ ), DDA [12] can achieve the feasible solution for all simulated cases. But, with increase in the SINR target and the network size, the feasibility rate of DDA [12] drops significantly. For example, in multicell network $1 \mathrm{~b}$ for SINR target $\gamma_{l}=15 \mathrm{~dB}$, DDA [12] is not able to find a feasible solution for any of the fading realization.

Figure 5 shows the average sum power versus iteration for multicell network 1a. The SINR target $\gamma_{l}$ is set to 15
$\mathrm{dB}$ for all $l \in \mathcal{L}$. For a comparison, we consider centralized algorithm [16, Section IV] and DDA [12]. DDA [12] plots are drawn for the subgradient step size $\alpha=10,50$. For a fair comparison of Algorithm 1, DDA [12], and the centralized algorithm [16, Section IV], the plots are drawn for the fading realizations that are feasible for all considered algorithms. Results show that the convergence speed of proposed Algorithm 1 compared with DDA [12] is fast and can achieves the centralized solution in less than ten iterations.

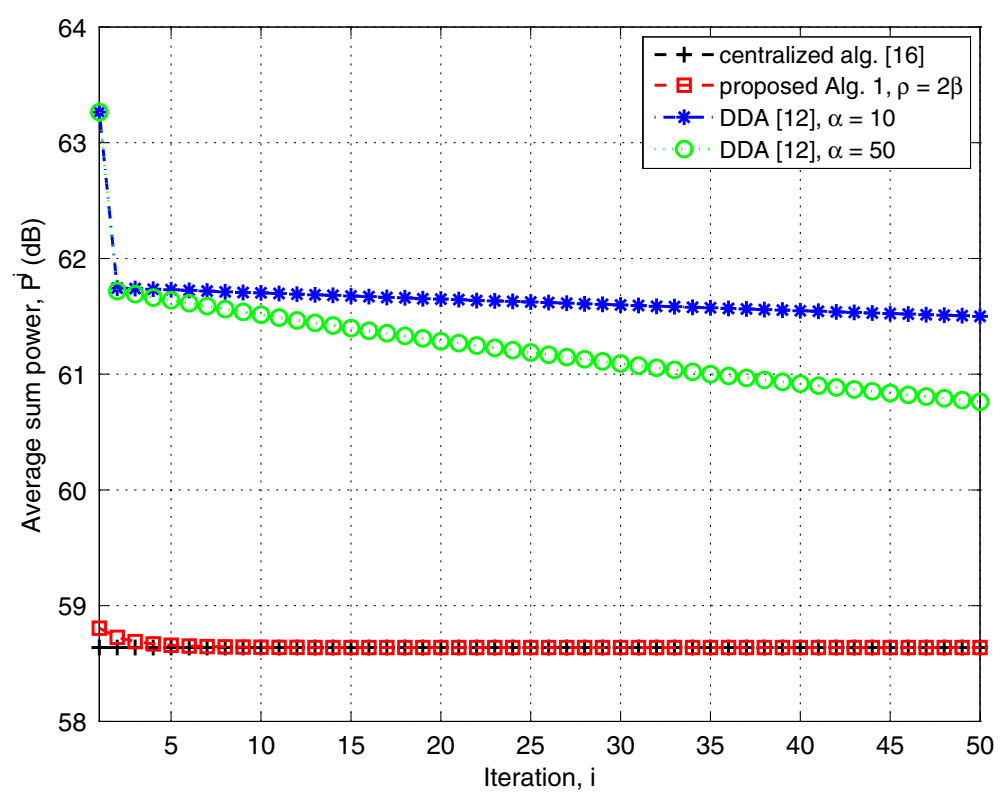

Figure 5 Multicell network 1: Average sum power versus iteration. Multicell network 1: Average sum power versus iteration for SINR target $\gamma_{l}=15 \mathrm{~dB}$ for all $/ \in \mathcal{L}$. 


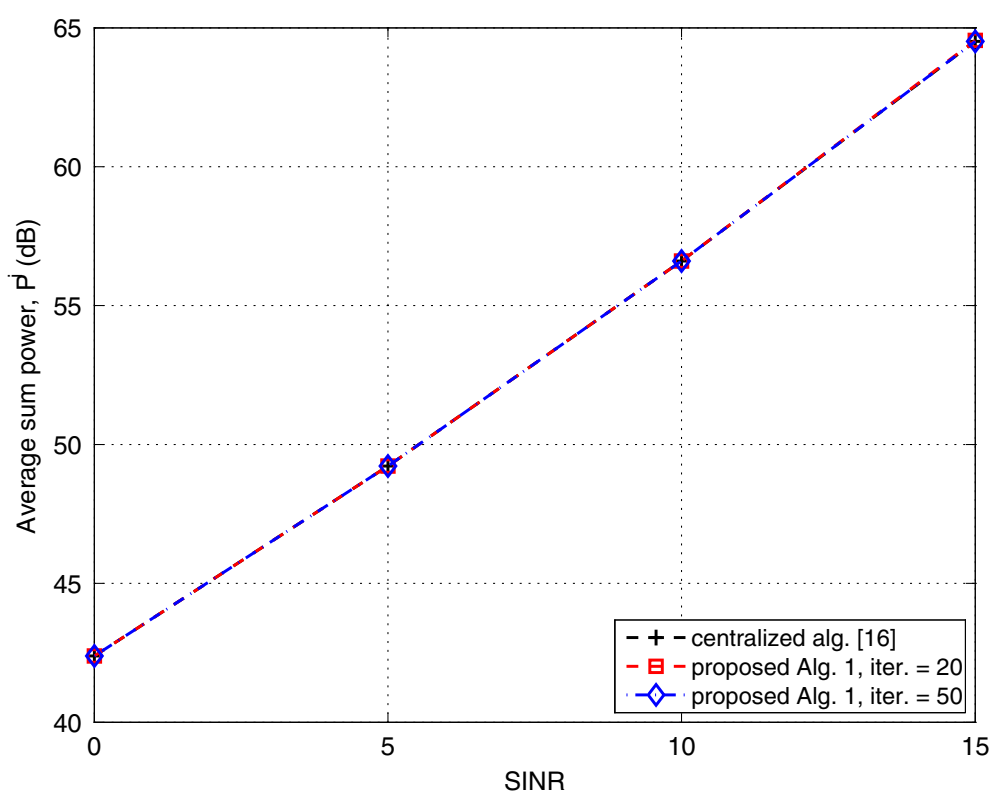

Figure 6 Multicell network 2: Average sum power versus SINR. Multicell network 2: Average sum power versus SINR for $\rho=2 \beta$.

Figure 6 shows the average sum power versus SINR target for multicell network $1 \mathrm{~b}$. For a comparison, we consider centralized algorithm [16, Section IV]. To note a fair progress of the proposed algorithm for a wide SINR target values, each curve is averaged for the fading realizations that are feasible for all the SINR values. Plots are drawn for the average sum power at iteration number 10 and 50 . Results show that the proposed Algorithm 1 can achieve the centralized solution over the wide rage of SINR target values.
We next evaluate the performance of Algorithm 3 for SINR balancing problem (P2). We, first, consider single fading realization and run the algorithm for both networks shown in Figure 1. As a benchmark, we consider centralized optimal algorithm proposed in [16, Section V]. In the simulation, we set SNR $=5 \mathrm{~dB}$, and for Algorithm 2, we set $\epsilon=0.1$, and $\alpha_{n}^{\max }=2 \times 10^{0.1 \times \mathrm{SNR}}$ for all $n \in \mathcal{N}$. Plots are drawn for $\rho=0.5,1,2$.

Figure 7 shows the progress of the global variable $\gamma$ by iteration. Note that the global variable $\gamma$ is the average of

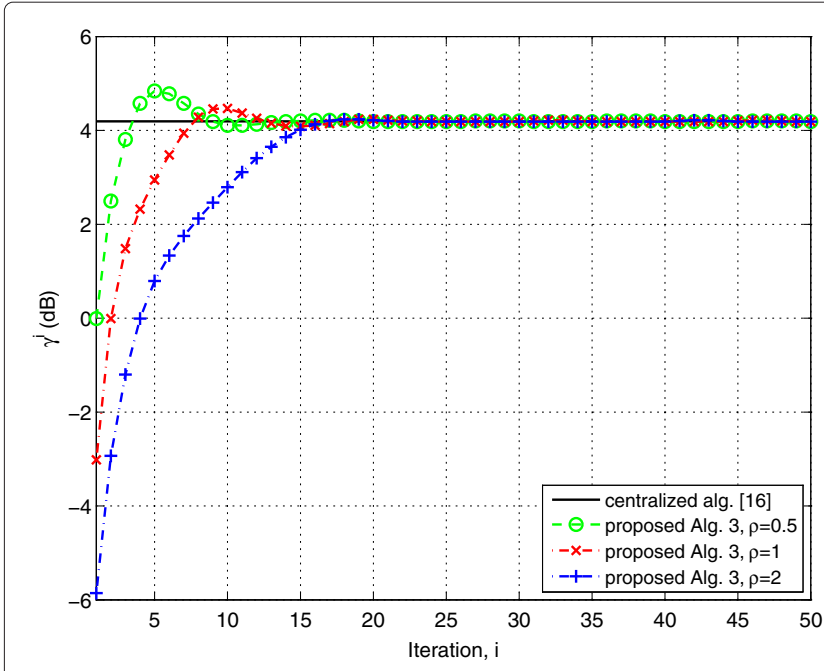

(a)

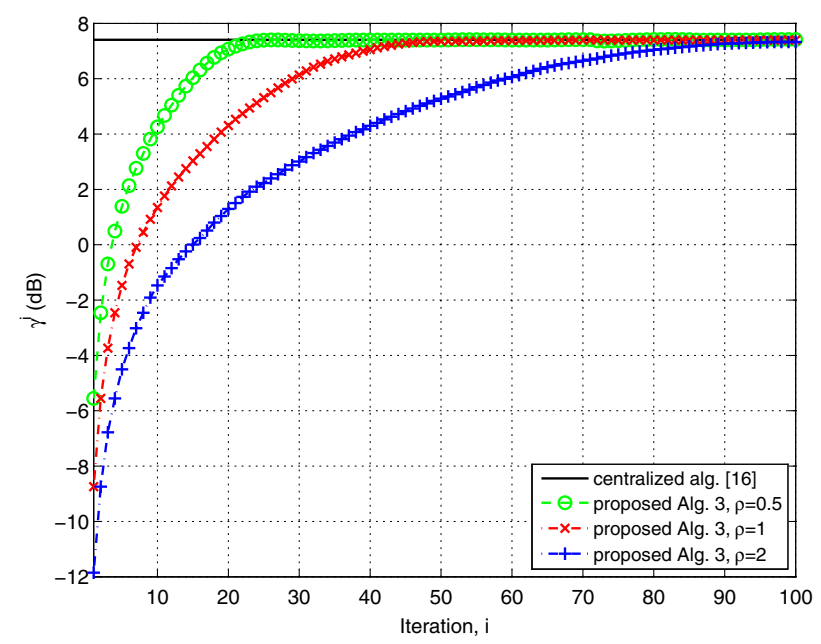

(b)

Figure 7 Evolution of average SINR for different algorithm parameter $\boldsymbol{\rho}$. Progress of global variable $\gamma$ for SNR $=5 \mathrm{~dB}$ : (a) Multicell network 1; (b) Multicell network 2. 


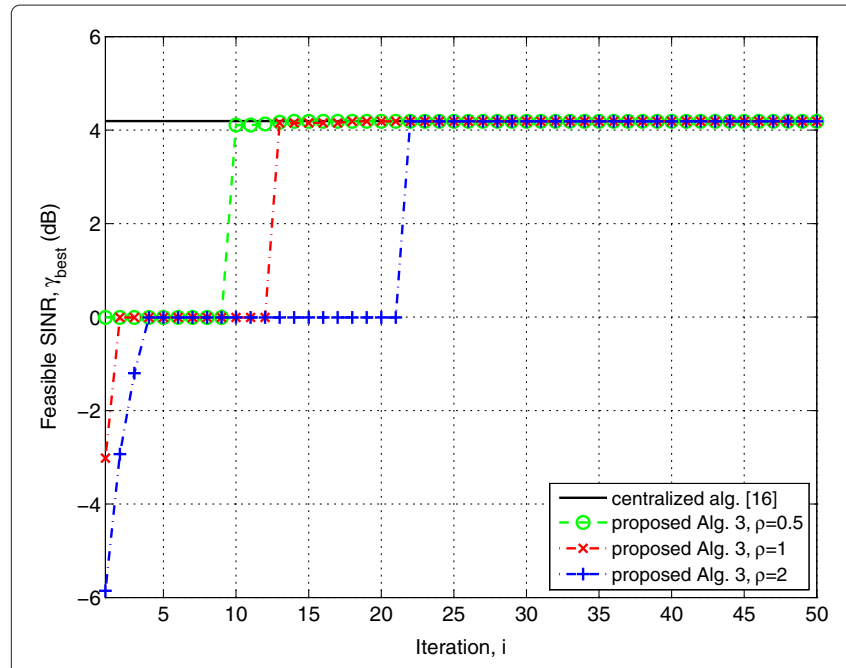

(a)

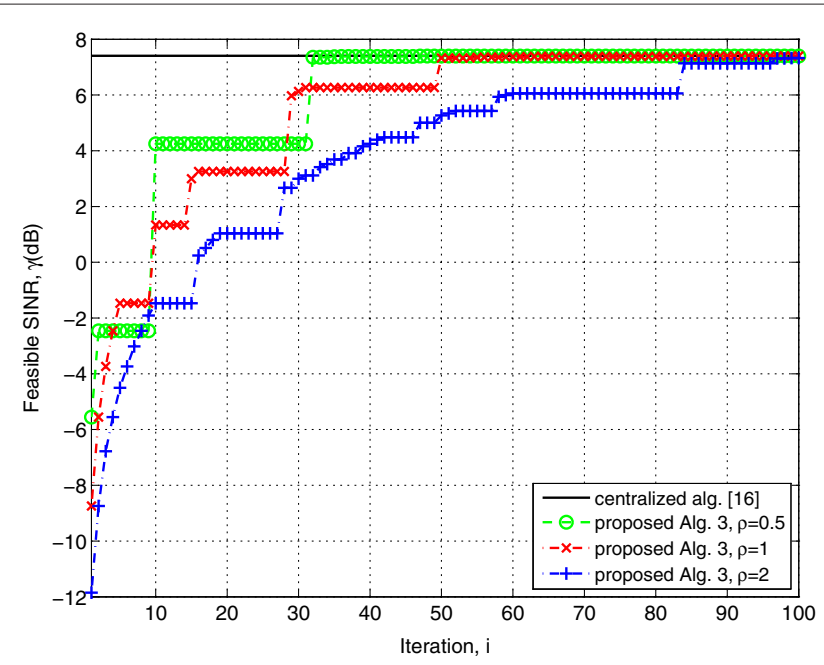

(b)

Figure 8 Evolution of an average best SINR, that is feasible for all BSs. Feasible SINR $\gamma_{\text {best }}^{i}$ versus iteration for SNR $=5 \mathrm{~dB}$ : (a) Multicell network 1; (b) Multicell network 2.

SINR values $\left\{\alpha_{n}\right\}_{n \in \mathcal{N}}$ that is obtained independently by all $N$ BSs (see (51)). Results show that for all considered values of $\rho$, Algorithm 3 can obtain SINR $\gamma$ that converges to the optimal centralized solution. Since $\gamma$ is the average of the SINR values obtained independently in all $N$ BSs, the intermediate values of $\gamma$ may not be feasible for all BSs before the algorithm converges. For example, the value of $\gamma$ for $\rho=0.5$ is clearly infeasible at the iteration step $i=\{4,5,6,7,8\}$ in Figure $7 \mathrm{a}$. Therefore, to illustrate the convergence of feasible $\gamma$, we define the following metric

$$
\gamma_{\text {best }}^{i}=\max _{t=1, \ldots, i}\left\{\gamma_{\text {feas }}^{t}\right\}
$$

where $\gamma_{\text {best }}^{i}$ is the best feasible SINR value at $i$ th iteration, and $\gamma_{\text {feas }}^{t}$ is the feasible SINR at $t$ th iteration (53). Figure 8 shows the behavior of $\gamma_{\text {best }}^{i}$ by iteration. Results show that Algorithm 3 can obtain the feasible values of $\gamma$ that converges to the centralized solution. For example, with $\rho=0.5$, the algorithm converges to the centralized solution in just tenth iterations in Figure 8a.

Figure 9 shows the SINR $\gamma_{\text {best }}^{i}$ for different SNR values $^{\circ}$. Each curve is averaged over 300 fading realizations. In the simulation, penalty parameter $\rho$ is set to 0.5 . Plots are drawn for the SINR obtained at iteration number 20, 30, and 50 of Algorithm 3. Results show that the proposed

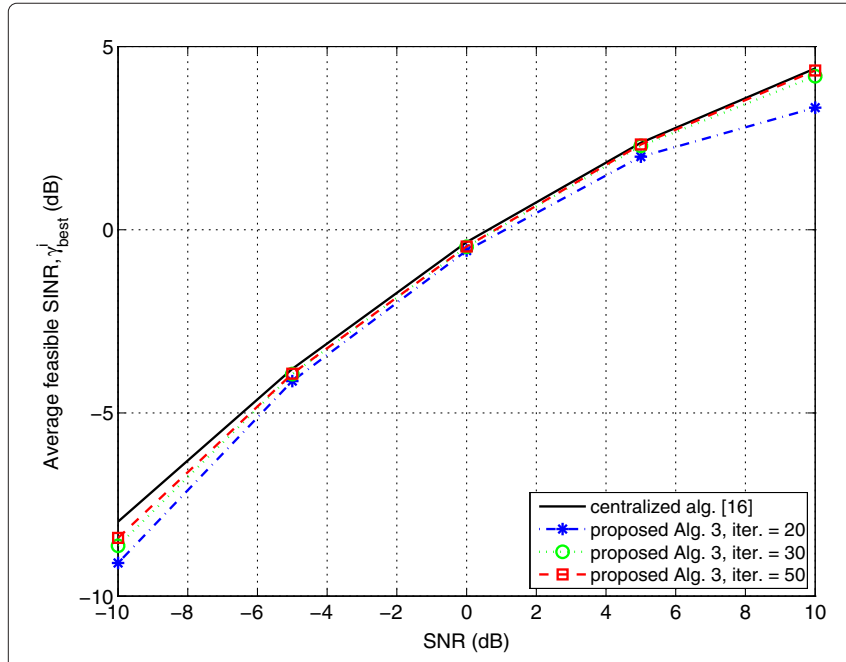

(a)

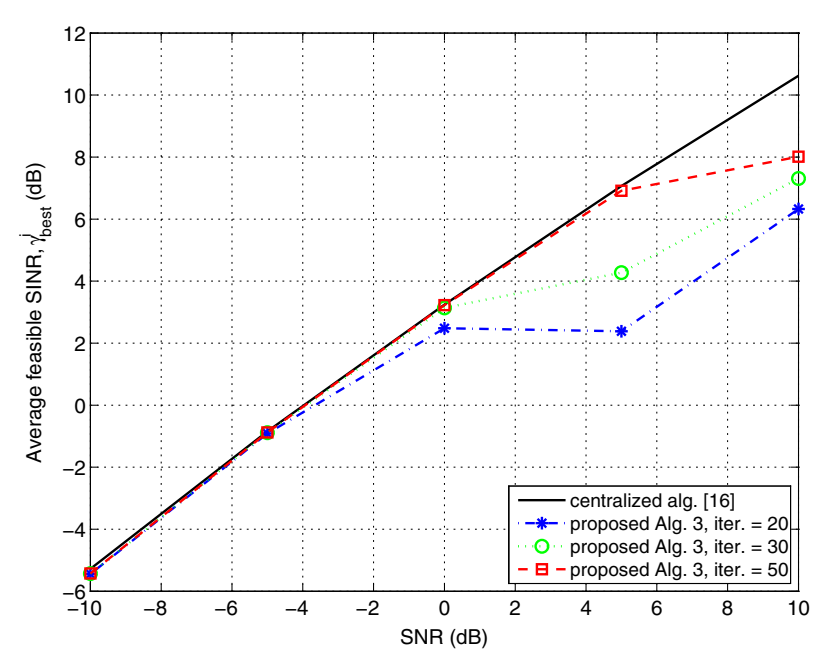

(b)

Figure 9 Feasible SINR versus SNR. Feasible SINR $\gamma_{\text {best }}^{i}$ versus SNR for $\rho=0.5$ : (a) Multicell network 1; (b) Multicell network 2. 
Algorithm 3 can achieve close to the centralized solution over the wide range of SNR values without any tuning of $\rho$.

\section{Conclusion}

We have provided distributed algorithms for the radio resource allocation problems in multicell downlink multiinput single-output systems. Specifically, we have considered two optimization problems: P1 - minimization of the total transmission power subject to signal-tointerference-plus-noise ratio (SINR) constraints of each user, and P2 - SINR balancing subject to total transmit power constraint of BSs. We have proposed consensusbased distributed algorithms, and the fast solution method via alternating direction method of multipliers. First, we have derived a distributed algorithm for problem P1. Then, in conjunction with the bracketing method, the algorithm is extended for problem P2. Numerical results show that the proposed distributed algorithms converge very fast to the optimal centralized solution.

\section{Endnotes}

a Similar assumptions are made, e.g., in [36] in the context of arbitrary wireless networks.

b The value of $R_{\text {int }}$ is chosen such that the power of the interference term is below the noise level, and this commonly used approximation is made to avoid unnecessary coordinations between distant BSs. The appropriate value of $R_{\text {int }}$ can be chosen to trade off between the required backhaul signaling and the optimality of the solution. The effect of nonzero $z_{n l}$ terms can be accurately modeled by changing the statistical characteristics of noise $n_{l}$ at $\operatorname{rec}(l)$. However, those issues are extraneous to the main focus of the paper.

${ }^{\mathrm{c}}$ In problem (4) and (5), the set $\left\{z_{n l}\right\}_{l \in \mathcal{L}_{\text {int }}, n \in \mathcal{N}_{\text {int }}(l)}$ is a collection of $z_{n l}$ for which the $l$ th user is inside the interference region of BS $n$. Thus, the constrained for unconsidered out-of-cell interference term (i.e., $z_{n l}^{2}=0$ ) for $l$ th user that is outside the interference region of BS $n$ is dropped in problem (4) and (5).

d A more general SINR balancing problem which can set priority of users (keeping the SINR values of data stream to a fixed ratios) $[6$, Section IV-C] can be formulated. To simplify the presentation, we consider maximization of the minimum SINR. Note that the proposed decentralized method can be easily generalized to the more general problem considered in

[6, Section IV-C].

e Note that $\mathcal{L}_{\text {int }}(n) \subseteq \mathcal{L}(n)$. Hence, $\operatorname{tran}(l)=n$ for all $l \in \mathcal{L}_{\text {int }}(n)$.

f To simplify the presentation, here we have used a slight abuse of notation, i.e., we have considered that the sets in (12) are ordered.

g Let $\left\{u_{k, n l}\right\}_{k \in\{n, \operatorname{tran}(l)\}, l \in \mathcal{L}_{\text {int }}, n \in \mathcal{N}_{\text {int }}(l)}$ be the dual variables associated with the equality constraints of problem (8), then by following steps (10) to (12), one can easily express $\mathbf{u}_{n}=\left\{\left\{u_{n, n l}\right\}_{l \in \mathcal{I}_{\text {int }}(n)},\left\{u_{n, b l}\right\}_{l \in \mathcal{L}_{\text {int }}(n), b \in \mathcal{N}_{\text {int }}(l)}\right\}, n \in \mathcal{N}$.

h Variable $z_{n l}$ (component of $\mathbf{z}_{n}$ ) couples two local variables $x_{n, n l}$ (component of $\mathbf{x}_{n}$ ) and $x_{\operatorname{tran}(l), n l}$ (component of $\mathbf{x}_{\operatorname{tran}(l)}$ ). Hence, in step (19) to update $z_{n l}$ coordination between $\mathrm{BS} n$ and $\mathrm{BS} \operatorname{tran}(l)$ is required.

${ }^{i}$ For convenience, we can combine the linear and quadratic terms of problem (21) as $\mathbf{u}_{n}^{i T}\left(\mathbf{x}_{n}-\mathbf{z}_{n}^{i}\right)+$ $\frac{\rho}{2}\left\|\mathbf{x}_{n}-\mathbf{z}_{n}^{i}\right\|_{2}^{2}=\frac{\rho}{2}\left\|\mathbf{x}_{n}-\mathbf{z}_{n}^{i}+\mathbf{v}_{n}^{i}\right\|_{2}^{2}-\frac{\rho}{2}\left\|\mathbf{v}_{n}^{i}\right\|_{2}^{2}$.

j Note that $\left\{\mathbf{u}_{n}\right\}_{n \in \mathcal{N}}$ are the dual variables associate with the consistency constraints of problem (16). By following steps (10) to (12), we can easily show $\mathbf{u}_{n}=\left\{\left\{u_{n, n l}\right\}_{l \in \mathcal{I}_{\text {int }}(n)}\right.$, $\left.\left\{u_{n, b l}\right\}_{l \in \mathcal{L}_{\text {int }}(n), b \in \mathcal{N}_{\text {int }}(l)}\right\}, n \in \mathcal{N}$.

${ }^{\mathrm{k}}$ Note that (20) in the components of $\mathbf{u}_{n}, \mathbf{x}_{n}$, and $\mathbf{z}_{n}$ can be expressed as $u_{k, n l}^{i+1}=u_{k, n l}^{i}+\rho\left(x_{k, n l}^{i+1}-z_{n l}^{i+1}\right)$ for all $k \in\{n, \operatorname{tran}(l)\}, l \in \mathcal{L}_{\text {int }}, n \in \mathcal{N}_{\text {int }}(l)$.

${ }^{1}$ In ADMM algorithm, standard stopping criteria is to check primal and dual residuals [21]. However, it is often the case that ADMM can produce acceptable results of practical use within a few tens of iteration [21]. As, finite number of iteration is more favorable for practical implementation, we adopt fixed number of iteration to stop the algorithm.

$m$ For convenience we can combine the terms in problem (40) as a) $\mathbf{u}_{n}^{i T}\left(\mathbf{x}_{n}-\mathbf{z}_{n}^{i}\right)+\frac{\rho}{2}\left\|\mathbf{x}_{n}-\mathbf{z}_{n}^{i}\right\|_{2}^{2}=$ $\frac{\rho}{2}\left\|\mathbf{x}_{n}-\mathbf{z}_{n}^{i}+\mathbf{v}_{n}^{i}\right\|_{2}^{2}-\frac{\rho}{2}\left\|\mathbf{v}_{n}^{i}\right\|_{2}^{2}$ and b) $v_{n}^{i}\left(\alpha_{n}-\gamma^{i}\right)+$ $\frac{\rho}{2}\left(\alpha_{n}-\gamma^{i}\right)^{2}=\frac{\rho}{2}\left(\alpha_{n}-\gamma^{i}+\lambda_{n}^{i}\right)^{2}-\frac{\rho}{2}\left(\lambda_{n}^{i}\right)^{2}$.

${ }^{\mathrm{n}}$ In ADMM algorithm, standard stopping criteria is to check primal and dual residuals [21]. However, it is often the case that ADMM can produce acceptable results of practical use within a few tens of iteration [21]. As, finite number of iteration is favorable for practical implementation, we adopt fixed number of iteration to stop the algorithm.

o For fixed radius $R_{\mathrm{BS}}$ in Figure 1, different SNRs (i.e., different $\left.\operatorname{SNR}\left(R_{\mathrm{BS}}\right)\right)$ are obtained by changing $\frac{p_{0}^{\max }}{\sigma^{2}}$ in (54).

PThe interval $\left[0, \alpha_{n}^{\max }\right]$ denotes the range of feasible $\alpha_{n}$ for problem (46).

\section{Appendices}

\section{Appendix 1}

In this appendix, we propose the bracketing method $[30,31]$ to solve problem (44). Let us start by combining the second (linear) and third (quadratic) terms of (45) as

$$
\begin{aligned}
p\left(\alpha_{n}\right)= & \tilde{p}\left(\alpha_{n}\right)+\frac{\rho}{2}\left(\alpha_{n}-\gamma^{i}+\lambda_{n}^{i}-\frac{1}{\rho N}\right)^{2} \\
& -\frac{1}{N}\left(\gamma^{i}-\lambda_{n}^{i}+\frac{1}{2}\right) .
\end{aligned}
$$

Without loss of generality, let us drop the constant term of (57) and simplify it as

$$
p\left(\alpha_{n}\right)=\tilde{p}\left(\alpha_{n}\right)+\frac{\rho}{2}\left(\alpha_{n}-\theta\right)^{2},
$$


where $\theta=\gamma^{i}-\lambda_{n}^{i}+\frac{1}{\rho N}$.

Note that the optimal value $\tilde{p}\left(\alpha_{n}\right)$ is nondecreasing function of $\alpha_{n} \in\left[0, \alpha_{n}^{\max }\right]^{\mathrm{p}}$. To see that, let $\mathcal{P}_{i}$ and $\mathcal{P}_{j}$ be the feasible set of problem (46) for $\alpha_{n}=\alpha_{n}^{i}$ and $\alpha_{n}=$ $\alpha_{n}^{j}$, respectively. If $\alpha_{n}^{j} \geq \alpha_{n}^{i}$, then it is easy to see that $\mathcal{P}_{j} \subseteq \mathcal{P}_{i}$. Hence, the optimal value $\tilde{p}\left(\alpha_{n}^{j}\right) \geq \tilde{p}\left(\alpha_{n}^{i}\right)$ for all $\alpha_{n}^{j} \geq \alpha_{n}^{i}$ and $\alpha_{n}^{i}, \alpha_{n}^{j} \in\left[0, \alpha_{n}^{\max }\right]$. Furthermore, there exists a partition of $\left[0, \alpha_{n}^{\max }\right]$ as $[0, \phi] \cup\left[\phi, \alpha_{n}^{\max }\right]$ such that

$$
\tilde{p}\left(\alpha_{n}\right)=c, \quad \alpha_{n} \in[0, \phi],
$$

where $c$ is the optimal solution of problem (46) for $\alpha_{n}=0$.

Next, we propose to use bracketing method $[30,31]$ to find the infimum of function $p\left(\alpha_{n}\right)$ on the interval $\alpha_{n} \in$ $\left[0, \alpha_{n}^{\max }\right]$. First, in Lemma 1 , we show that the function $p\left(\alpha_{n}\right)$ is a unimodal function on the interval $\alpha_{n} \in\left[0, \alpha_{n}^{\max }\right]$ for the condition: C) $\theta \leq \phi$.

Lemma 1. The function $p\left(\alpha_{n}\right)$,

$$
p\left(\alpha_{n}\right)=\tilde{p}\left(\alpha_{n}\right)+\frac{\rho}{2}\left(\alpha_{n}-\theta\right)^{2},
$$

is a unimodal function on the interval $\alpha_{n} \in\left[0, \alpha_{n}^{\max }\right]$ for the condition $C$.

\section{Proof:}

1. For the case $\theta \leq 0$, the proof is trivial, since $p\left(\alpha_{n}\right)$ is a sum of two increasing functions on the interval $\alpha_{n} \in\left[0, \alpha_{n}^{\max }\right]$.

2. For the case $\theta>0$, let us partition $\left[0, \alpha_{n}^{\max }\right]$ as $[0, \theta] \cup\left[\theta, \alpha_{n}^{\max }\right]$. On the interval $\alpha_{n} \in[0, \theta]$, the function $\tilde{p}\left(\alpha_{n}\right)$ takes a constant value $c$. On the interval $\alpha_{n} \in\left[\theta, \alpha_{n}^{\max }\right]$, the function $\tilde{p}\left(\alpha_{n}\right)$ is a nondecreasing function. Hence, the function $p\left(\alpha_{n}\right)$ is a sum of affine and convex functions on the interval $[0, \theta]$, and a sum of nondecreasing and increasing functions on the interval $\left[\theta, \alpha_{n}^{\max }\right]$. Thus, the function $p\left(\alpha_{n}\right)$ is a unimodal function.

Lemma 1 implies that for the condition $C$ (i.e., $\theta \leq$ $\phi)$, the infimum of the function $p\left(\alpha_{n}\right)$ can be obtained optimally by using bracketing method [30,31].

For the case condition $C$ is not satisfied (i.e., $\phi \leq \theta$ ), let us partition $\left[0, \alpha_{n}^{\max }\right]$ as $[0, \phi] \cup[\phi, \theta] \cup\left[\theta, \alpha_{n}^{\max }\right]$. On the interval $\alpha_{n} \in[0, \phi]$, the function $p\left(\alpha_{n}\right)$ is a decreasing function (since $\tilde{p}\left(\alpha_{n}\right)$ takes a constant value $c$, and $\left(\alpha_{n}-\theta\right)^{2}$ is a decreasing function). On the interval $\alpha_{n} \in$ $\left[\theta, \alpha_{n}^{\max }\right]$, the function $p\left(\alpha_{n}\right)$ is an increasing function (since $\tilde{p}\left(\alpha_{n}\right)$ is nondecreasing function and $\left(\alpha_{n}-\theta\right)^{2}$ is increasing function). On the interval $\alpha_{n} \in[\phi, \theta]$, analytically expressing the curvature of $p\left(\alpha_{n}\right)$ is difficult, since the curvature of function $\tilde{p}\left(\alpha_{n}\right)$ depends on the numerical parameters. This implies that for the case $\phi \leq \theta$, the infimum of the function $p\left(\alpha_{n}\right)$ lies on the interval $[\phi, \theta]$, i.e.,

$$
\underset{\alpha_{n} \in\left[0, \alpha_{n}^{\max }\right]}{\arg \min } p\left(\alpha_{n}\right) \in[\phi, \theta] .
$$

Thus in the case $\phi \leq \theta$ (i.e., if condition $\mathrm{C}$ is not satisfied), the solution of problem (61) obtained by using bracketing method [30,31] lies at most $(\theta-\phi)$ away from the optimal solution. However, in all of our numerical simulations, we have always noted that the function $p\left(\alpha_{n}\right)$ is a unimodal function. In that case, problem (61) is solved optimally by bracketing method [30,31]. Moreover, the convergence of the proposed Algorithm 3 (see numerical example, Section 5) to the centralized solution shows that bracketing method can be used to solve problem (44).

\section{Appendix 2}

The ADMM method is guaranteed to converge for all values of its penalty parameter $\rho$ [21]. However, the rate of convergence of ADMM algorithm is sensitive to the choice of the penalty parameter $\rho$. In practice, the ADMM penalty parameter $\rho$ is either tuned empirically for each specific application, or set equal to 1 by normalizing the problem data set [21, Chapter 11]. Note that in Algorithm 1, to solve the local variable update (22), we can normalize the problem data (i.e., sum power $\left.\left(\sum_{l \in \mathcal{L}(n)}\left\|\mathbf{m}_{l}\right\|_{2}^{2}\right)\right)$ by normalizing factor $\beta_{n}>0$ and set $\rho=1$, which is equivalent to set $\rho=\beta_{n}$ in Algorithm 1 , if the problem data (i.e., sum power $\left(\sum_{l \in \mathcal{L}(n)}\left\|\mathbf{m}_{l}\right\|_{2}^{2}\right)$ ) is not normalized. To elaborate further, let us express equivalently the local variable update (22) as

$$
\begin{aligned}
\operatorname{minimize} & \frac{1}{\beta_{n}}\left(\sum_{l \in \mathcal{L}(n)}\left\|\mathbf{m}_{l}\right\|_{2}^{2}\right)+\frac{\rho}{2}\left\|\mathbf{x}_{n}-\mathbf{z}_{n}^{i}+\mathbf{v}_{n}^{i}\right\|_{2}^{2} \\
\text { subject to } & {\left[\begin{array}{c}
\sqrt{1+\frac{1}{\gamma_{l}}} \mathbf{h}_{l l}^{\mathrm{H}} \mathbf{m}_{l} \\
\mathbf{M}_{n}^{\mathrm{H}} \mathbf{h}_{l l} \\
\tilde{\mathbf{x}}_{l} \\
\sigma_{l}
\end{array}\right]{ }_{\operatorname{SOC} 0, \quad l \in \mathcal{L}(n)} 0 } \\
& {\left[\begin{array}{c}
x_{n, n l} \\
\mathbf{M}_{n}^{\mathrm{H}} \mathbf{h}_{j l}
\end{array}\right] \succeq_{\text {SOC } 0, \quad l \in \mathcal{I}_{\text {int }}(n)} }
\end{aligned}
$$

with variables $\mathbf{M}_{n}=\left[\mathbf{m}_{l}\right]_{l \in \mathcal{L}(n)}$ and $\mathbf{x}_{n}$, where $\beta_{n}>0$ is the normalizing factor, $\tilde{\mathbf{x}}_{l}=\left\{x_{n, b l}\right\}_{b \in \mathcal{N}_{\text {int }}(l)}$ is a subset of $\mathbf{x}_{n}$ (see (12)), the matrix $\mathbf{h}_{j l}$ in the second set of constraints denotes the channel from BS $n$ to link $l$ (i.e., the index $j$ in the third set of constraints denotes an arbitrary link in $\mathcal{L}(n)$ ), and the notation $\succeq$ sOC denotes the 
generalized inequalities with respect to the second-order cone $[16,19]$.

For problem (62), the optimal choice of $\beta_{n}$ is $\sum_{l \in \mathcal{L}(n)}\left\|\mathbf{m}_{l}^{\star}\right\|_{2}^{2}$. However, before the convergence of Algorithm 1, we do not have optimal beamformers (i.e., $\left.\left\{\mathbf{m}_{l}^{\star}\right\}_{l \in \mathcal{L}(n)}\right)$. Thus, in our simulation, to estimate $\beta_{n}$, we ignore the interference and noise terms and find beamforming vector $\tilde{\mathbf{m}}_{l}$ that achieves the required SINR threshold $\gamma_{l}$, which can be expressed as

$$
\tilde{\mathbf{m}}_{l}=\left(10^{0.1 \times \gamma_{l}}\right) /\left\|\mathbf{h}_{l l}\right\|_{2}^{2}, \quad l \in \mathcal{L}(n)
$$

Hence, the normalizing factor $\beta_{n}$ for problem (62) can be written as

$$
\begin{aligned}
\beta_{n} & =\sum_{l \in \mathcal{L}(n)} \tilde{\mathbf{m}}_{l} \\
& =\sum_{l \in \mathcal{L}(n)}\left(10^{0.1 \times \gamma_{l}}\right) /\left\|\mathbf{h}_{l l}\right\|_{2}^{2} .
\end{aligned}
$$

Furthermore, we find $\beta=\max _{n \in \mathcal{N}}\left\{\beta_{n}\right\}$, and set $\rho=\beta$ for Algorithm 1.

\section{Competing interests}

The authors declare that they have no competing interests.

\section{Acknowledgements}

This research was supported by the Finnish Funding Agency for Technology and Innovation (Tekes), Nokia Solutions and Networks, Anite Telecoms, Renesas Mobile Europe, and Elektrobit.

\section{Received: 14 April 2013 Accepted: 16 December 2013}

Published: 4 January 2014

\section{References}

1. M Bengtsson, B Ottersten, Optimal Downlink Beamforming Using Semidefinite Optimization, in Proceedings of the Annual Allerton Conference on Communications, Control, and Computing, (Urbana-Champaign IL, 1999), pp. 987-996

2. F Rashid-Farrokhi, KJR Liu, L Tassiulas, Transmit beamforming and power control for cellular wireless systems. IEEE J. Select. Areas Commun. 16(8), 1437-1450 (1998)

3. E Visotsky, U Madhow, vol. 1, Optimum Beamforming Using Transmit Antenna Arrays, in Proceedings of the IEEE Vehicular Technology Conference, (Houston, TX, 1999), pp. 851-856

4. Q Shi, M Razaviyayn, M Hong, ZQ Luo, SINR constrained beamforming for a MIMO multi-user downlink system, in Proceedings of the Annual Asilomar Conference on Signals, Systems and Computers, (Pacific Grove, CA, 2012), pp. 1991-1995

5. KK Wong, G Zheng, TS Ng, vol. 1, Convergence analysis of downlink MIMO antenna systems using second-order cone programming, in Proceedings of the IEEE Vehicular Technology Conference, (Dallas, 2005), pp. 492-496

6. M Codreanu, A Tölli, M Juntti, M Latva-aho, Joint design of Tx-Rx beamformers in MIMO Downlink channel. IEEE Trans. Signal Process. 55(9), 4639-4655 (2007)

7. M Schubert, $\mathrm{H}$ Boche, Solution of the Multiuser downlink beamforming problem with individual SINR constraints. IEEE Trans. Vehic. Technol. $\mathbf{5 3}$ 18-28 (2004)

8. H Boche, M Schubert, Optimal multi-user interference balancing using transmit beamforming. Wireless Pers. Commun., Kluwer Acad. Publishers. 26(4), 305-324 (2003)
9. M Schubert, $\mathrm{H}$ Boche, Comparison of $\ell_{\infty}$-norm and $\ell_{1}$-norm optimization criteria for SIR-balanced multi-user beamforming. Elsevier Signal Process. 84(2), 367-378 (2004)

10. A Tolli, M Codreanu, M Juntti, Minimum SINR maximization for multiuse MIMO downlink with per BS power constraints, in Proceedings of the IEEE Global Telecommunication Conference, (Kowloon, 2007), pp. 1144-1149

11. HDW Yu, Coordinated beamforming for the multicell multi-antenna wireless system. IEEE Trans. Wireless Commun. 9(5), 1748-1759 (2010)

12. A Tölli, H Pennanen, P Komulainen, Decentralized minimum power multi-cell beamforming with limited backhaul signaling. IEEE Trans. Wireless Commun. 10(2), 570-580 (2011)

13. H Pennanen, A Tolli, A Latva-ahom, Decentralized coordinated downlink beamforming via primal decomposition. IEEE Signal Process. Lett. 18(11) 647-650 (2011)

14. D Nguyen, T Le-Ngoc, Multiuser downlink beamforming in multicell wireless systems: a game theoretical approach. IEEE Trans. Signal Process. 59(7), 3326-3338 (2011)

15. C Shen, T Chang, K Wang, Z Qiu, C Chi, Distributed robust multicell coordinated beamforming with imperfect CSI: an ADMM approach. IEEE Trans. Signal Process. 60(6), 2988-3003 (2012)

16. A Wiesel, YC Eldar, S Shamai, Linear precoding via conic optimization for fixed MIMO receivers. IEEE Trans. Signal Proc. 54, 161-176 (2006)

17. S Boyd, L Xiao, A Mutapcic, J Mattingley, Notes on decomposition methods: course reader for convex optimization II, Stanford 2007. [[Online]. Available: http://www.stanford.edu/class/ee364b/notes/ decomposition notes.pdf]. Accessed 01 March 2012

18. S Boyd, Subgradient methods 2007. [[Online]. Available: http://www stanford.edu/class/ee364b/lectures/subgrad_method_slides.pdf]. Accessed 01 March 2012

19. S Boyd, L Vandenberghe, Convex optimization (Cambridge University Press, Cambridge, 2004)

20. Y Huang, G Zheng, M Bengtsson, KK Wong, L Yang, B Ottersten, Distributed multicell beamforming with limited intercell coordination. IEEE Trans. Signal Process. 59(2), 728-738 (2011)

21. S Boyd, N Parikh, E Chu, B Peleato, J Eckstein, Distributed optimization and statistical learning via the alternating direction method of multipliers. Foundations Trends Mach. Learn. 3, 1-122 (2010)

22. J Yang, Y Zhang, Alternating direction algorithms for $\ell_{1}$-problems in compressive sensing. SIAM J. Sci. Comput. 33, 250-278 (2011)

23. M Figueiredo, J Bioucas-Dias, Restoration of poissonian images using alternating direction optimization. IEEE Trans. Image Process. 19(12) 3133-3145 (2010)

24. I Schizas, A Ribeiro, G Giannakis, Consensus in ad hoc WSNs with noisy links-part I: distributed estimation of deterministic signals. IEEE Trans. Signal Process. 56, 350-364 (2008)

25. M Leinonen, M Codreanu, M Juntti, Distributed consensus based joint resource and routing optimization in wireless sensor networks, in Proceedings of the Annual Asilomar Conference on Signals, Systems and Computers, (Pacific Grove, CA, 2012)

26. B He, X Yuan, On the $O(1 / n)$ convergence rate of the Douglas-Rachford alternating direction method. SIAM J. Numerical Anal. 50(2), 700-709 (2012)

27. J Mota, J Xavier, P Aguiar, M Puschel, Distributed ADMM for model predictive control and congestion control, in Proceedings of the IEEE International Conference on Decision and Control, (Maui, HI, 2012), pp. 5110-5115

28. W Deng, W Yin, On the global and linear convergence of the generalized alternating direction method of multipliers, in Rice CAAM tech report TR12-14 (2012)

29. M Hong, ZQ Luo, On the linear convergence of the alternating direction method of multipliers, in arXiv preprint arXiv:1208.3922, (2012)

30. JH Mathews, KK Fink, Numerical Methods Using Matlab, 4th edn (Prentice-Hall Inc., Upper Saddle River, 2004)

31. W Cheney, D Kincaid, Numerical Mathematics and Computing, 4th edn (International Thomson Publishing, Stamford, 1998)

32. S Joshi, M Codreanu, M Latva-Aho, Distributed resource allocation for MISO downlink systems via the alternating direction method of multipliers, in Proceedings of the Annual Asilomar Conference on Signals, Systems and Computers, (Pacific Grove, CA, 2012), pp. 488-493

33. DP Bertsekas, Constrained Optimization and Lagrange Multiplier Methods (Academic Press, Waltham, 1982) 
34. D Bertsekas, J Tsitsiklis, Parallel and Distributed Computation: Numerical Methods (Athena Scientific, Belmont, 1997)

35. A Kumar, D Manjunath, J Kuri, Wireless Networking (Elsevier Inc., Burlington, 2008)

36. P Gupta, PR Kumar, The capacity of wireless networks. IEEE Trans. Inform. Theory 46(2), 388-404 (2000)

doi:10.1186/1687-1499-2014-1

Cite this article as: Joshi et al.: Distributed resource allocation for MISO downlink systems via the alternating direction method of multipliers. EURASIP Journal on Wireless Communications and Networking 2014 2014:1.

\section{Submit your manuscript to a SpringerOpen ${ }^{\odot}$ journal and benefit from:}

- Convenient online submission

- Rigorous peer review

- Immediate publication on acceptance

- Open access: articles freely available online

- High visibility within the field

- Retaining the copyright to your article 